\title{
Un percorso integrato di matematica e italiano in continuità dalla scuola dell'infanzia alla scuola secondaria di primo grado
}

\section{An integrated experience on mathematics and Italian language in continuity from kindergarten to lower secondary school}

\author{
Lorella Campolucci e Danila Maori \\ Gruppo MiR, Matematica in Rete - Corinaldo (Ancona), Italia ${ }^{1}$ \\ Rete di Istituti Comprensivi - capofila Istituto Comprensivo Corinaldo \\ 凶lorella.campolucci@alice.it, danilamaori@libero.it
}

Sunto / In questo articolo si raccontano alcune esperienze realizzate da un gruppo di docenti di scuola dell'infanzia, primaria e secondaria di primo grado basate su percorsi interdisciplinari di matematica e italiano. Le esperienze riguardano prevalentemente la lettura, la comprensione e la formulazione di problemi; l'uso di narrazioni per raccontare la matematica, introdurre nuovi concetti e far parlare dei concetti appresi; la ludolinguistica applicata alla matematica per un approccio positivo nei confronti dell'errore e per ampliare il lessico e le esperienze comunicative.

Parole chiave: Italmatica; problemi; ludolinguistica; narrazioni matematiche.

\begin{abstract}
This article describes some of the experiences carried out by a group of teachers of kindergarten, primary and lower secondary school based on interdisciplinary courses in Mathematics and Italian.

Experiences are mainly concerned with reading, understanding and formulating problems; the use of narratives to tell mathematics, introduce new concepts and make people talk about the concepts learned; the ludolinguistics applied to mathematics for a positive approach to error and to expand the vocabulary and communication experiences.
\end{abstract}

Keywords: Italmatica; problems; ludolinguistics; mathematical storytelling.

\footnotetext{
1. Tutte le attività sono state pensate, progettate e costruite all'interno del gruppo composto anche da: Aguzzi Cinzia, Alesi Catia, Amichetti Sonia, Anconetani Fabiana, Andreoli Alessandra, Angeletti Rossella, Antinucci Ombretta, Antognoni Angelo, Antenucci Melissa, Asaro Graziella, Baldelli Martina, Baldi Laura, Baldoni Rita, Bambini Cinzia, Bartoli Francesca, Battistelli M. Cristina, Battiston Eleonora, Benigni Stefania, Berardi David, Borioni Anna, Bossoletti Sonia, Bracceschi M. Angela, Bracci Tiziana, Brescini Tiziana, Brunner Alexandra, Brutti Paola, Bucci Cristina, Bucci Luigia, Buldrighini Monica, Burattini Carla, Burattini Danilo, Buschi Stefania, Buzi Paola, Caimmi M. Loretta, Camillini Simonetta, Campitelli Anna, Caragiulo Micaela, Casagrande Carolina, Catozzi Mariangiola, Ceccarelli Roberta, Ceciliani Loredana, Celani Katia, Cerioni Francesca, Cesaretti Cinzia, Chiodi Donatella, Cittadini Serena, Conti Silvia, Coppa Luana, De Marchi Roberta, Discepoli Elena, Dori Paola, Enache Carmen, Ercolani Francesca, Fabrizi Silvana, Favatà Elisena, Fiorani Ambra, Fioretti Federica, Formato Giulia, Frati Anna Maria, Gagliardini M. Luisa, Galli M. Teresa, Giaccaglia Silvia, Giaccoli Luciana, Giancamilli Simona, Gianuario Francesca, Genga Marta, Giorgi Catia, Girini Manuela, Giuliani Roberta, Granini Marta, Guerra Giuseppina, Guidi Armanda, Magnarelli Alessandra, Maiolatesi Tiziana, Mancinelli Barbara, Mancini Romina, Mancini Simona, Manoni Miriam, Marcelli Lorella, Marchetti Francesca, Mariotti Cristina, Mariotti Rosa, Mariotto Paola, Mattina Giuseppina, Mencarelli Giulia, Merli Cristiana, Montesi Raffaella, Morbidelli Elena, Moroni Mara, Mosconi Catia, Niccoli Silvia, Nobili Assuntina, Ortuso Lina, Paccazzocco Giovanna, Pacini Paola, Pallotta Claudia, Paoletti Sonia, Papalini Paola, Paradisi Giorgia, Paradisi Lorena, Patregnani Laura, Perini Angela, Perini Laura, Piciotti Donatella, Pierantoni Elisa, Pierantoni Serenella, Pierfederici Luca, Pierini Eralda, Pieroni Chiara, Pierpaoli Micaela, Pierucci Chiara, Pigliapoco Chiara, Pistelli Rossana, Poli Sara, Polverari Marina, Principi Cinzia, Rigante Teresa, Romagnoli Claudia, Romagnoli Daniela, Romagnoli Marianna, Romanini Cinzia, Ronconi Loretta, Rosa Elenia, Rossi Anna, Rossi Barbara, Rossolini Emilia, Rotatori Federica, Rugini Katia, Sani Anna Maria, Santinelli Elda Maria, Santini Gloria, Santori Marta, Severini Anna Maria, Silvestro Rosa, Spadoni Arianna, Sparacciari Valentina, Sparvoli Simonetta, Spinaci Cinzia, Stefanelli Susanna, Stefanini Margherita, Straccini Silvia, Tinti Claudia, Tiranti Laura, Tomassoni Monica, Tommassetti Angela, Trillini Catia, Zappatori Verusca, Zocchi Barbara, Zuccari Loredana.
}

(C) 2021 Campolucci Lorella e Maori Danila. Questo è un articolo Open Access, sottoposto a un processo di revisione tra pari a doppio cieco, pubblicato dal Centro competenze didattica della matematica e dal Servizio comunicazione del DFA-SUPSI in collaborazione con il DECS. L'articolo è distribuito sotto i termini della Licenza Creative Commons Attribuzione 4.0 Internazionale (CC BY 4.0) che permette di usare, condividere e modificare l'articolo su qualsiasi mezzo a patto che l'autore e la fonte originale siano citati. 


\section{Presentazione}

"Italmatica - Percorsi integrati di matematica e italiano" è un progetto realizzato dal gruppo Matematica in Rete (MiR), un gruppo di lavoro/studio costituito da insegnanti di scuola dell'infanzia, primaria e secondaria di primo grado² di diversi Istituti Comprensivi della provincia di Ancona e di Pesaro/ Urbino, di cui è capofila I'Istituto Comprensivo di Corinaldo (Ancona). II gruppo si riunisce mensilmente, in questo periodo solo in digitale, allo scopo di fare formazione, progettare e realizzare proposte didattiche innovative e significative e favorire il dialogo tra gli insegnanti dei diversi livelli scolastici. Il gruppo è nato circa 20 anni fa sulla spinta di alcune di noi che, incuriosite dalle ricerche in didattica della matematica e affascinate dai seminari e dalle stimolanti attività nelle mostre del convegno nazionale "Incontri con la matematica" di Castel S. Pietro Terme (Bologna), si sono impegnate per attivare un primo corso di formazione con il gruppo di Ricerca e Sperimentazione in Didattica e Divulgazione della Matematica (RSDDM) di Bologna. Da allora sono iniziati i nostri contatti con il prof. D'Amore e la prof.ssa Sbaragli, abbiamo dato vita al gruppo, cominciato a incontrarci e a metterci in discussione non più a livello soltanto personale, ma anche come gruppo. Le difficoltà, abbastanza ovvie, generate dall'apertura al confronto collettivo mettendo a nudo i propri dubbi e le incertezze, sono state superate grazie all'entusiasmo per questo nuovo modo di lavorare insieme, di condividere successi e difficoltà, di confrontarsi sulla nostra formazione, sulle nostre competenze e sulle nostre esperienze, raccogliendo, documentando e condividendo le nostre produzioni.

Non abbiamo più abbandonato questa modalità di lavoro che alterna incontri di riflessione teorica con esperti e progettazione e realizzazione di attività didattiche in gruppo. È stata una vera conquista per le nostre scuole.

In tutti questi anni diversi docenti sono cambiati, ma molti sono ancora presenti e attivi, e abbiamo con continuità seguito formazioni con tanti esperti in didattica della matematica e altre discipline, i quali, sempre con grande generosità, si sono mostrati disponibili ad accogliere i nostri dubbi, le nostre paure, le nostre perplessità; abbiamo avuto la possibilità di approfondire i diversi nuclei di contenuto, condotto vari percorsi di riflessione sulle nostre convinzioni e sulla loro influenza nella didattica d'aula e abbiamo realizzato numerose esperienze concrete sul campo. Un lungo cammino di approfondimento che ci ha cambiati molto e ha trasformato la nostra didattica: oggi siamo più sensibili e più attenti al nostro modo di lavorare e siamo più disponibili a metterci in discussione e a ripensare alle attività che proponiamo. In tutti questi 20 anni il nostro costante mentore e il nostro tutor è stata la prof.ssa S. Sbaragli, che ci ha sempre coinvolto in progetti interessanti, ci ha tenuti uniti e sostenuti nella formazione, spronandoci a migliorare e a rivedere le nostre convinzioni. Certo, a volte, specialmente agli inizi, ci siamo sentiti un po' destabilizzati nei confronti delle nuove proposte, abbiamo perso i nostri punti di riferimento derivanti dalle routine scolastiche, le nostre ancore di sicurezza, ma proprio questo è stato il volano per intraprendere il cammino di revisione e di crescita professionale.

Negli ultimi anni la nostra attività si è concentrata sul legame imprescindibile che c'è tra la matematica e l'italiano. Sollecitati dalle ricerche condotte sui punti di contatto tra questi due mondi, ancora ritenuti distanti e separati tra loro (Demartini et al., 2017; Fornara \& Sbaragli, 2013), attraverso un percorso di formazione con le docenti S. Sbaragli e S. Demartini, abbiamo approfondito i legami tra le due discipline, sviluppato nuovi percorsi didattici e analizzato e ripensato le nostre proposte tramite un nuovo punto di vista. Nelle esperienze svolte abbiamo fatto emergere le aree di contatto, gli "intrecci", tra l'italiano e la matematica e abbiamo potuto verificare che una didattica combinata sollecita la curiosità, la creatività e il desiderio di sperimentare dei bambini e favorisce atteggiamenti di ricerca, progettazione, scoperta e invenzione. Queste sono le esperienze che cercheremo di descrivere in questo contributo.

2. La scuola dell'infanzia in Italia segue un percorso analogo alla scuola dell'infanzia in Canton Ticino. La scuola primaria in Italia dura cinque anni e corrisponde alla scuola elementare nel Canton Ticino. La scuola secondaria di primo grado in Italia dura tre anni e corrisponde ai primi tre anni di scuola media nel Canton Ticino. 


\title{
2 Approccio metodologico
}

Nelle nostre proposte cerchiamo sempre di usare strategie didattiche che mettano al centro dell'attenzione gli alunni e la costruzione delle loro competenze. Nella convinzione che ogni metodologia, da sola, presenti dei limiti, I'approccio adottato si basa sull'applicazione di diverse metodologie didattiche: la didattica laboratoriale per rendere gli allievi protagonisti attivi nella costruzione di oggetti e conoscenze; I'uso delle tecnologie per favorire la condivisione, I'astrazione, la comunicazione; il cooperative learning per creare occasioni di confronto, collaborazione e sostegno reciproco; il gioco, mediatore e produttore di conoscenze formidabile, per suscitare l'interesse e la motivazione a tutti i livelli scolastici. Come già accennato, negli ultimi anni a queste strategie si è unita la ricerca delle aree di incontro tra matematica e italiano e il lavoro in parallelo tra le due discipline, avviando così progetti interdisciplinari: abbiamo condotto riflessioni sulle strategie di lettura, di comprensione profonda, di scrittura e di revisione di testi di vario genere; abbiamo analizzato alcune tecniche di narrazione e sperimentato con maggiore profondità il ruolo della narrazione in ambito matematico, l'uso e la creazione di storie, di albi illustrati; abbiamo approfondito l'importanza delle parole e del loro significato nei vari contesti d'uso e la valenza dei giochi linguistici applicati alla matematica. Questo percorso ci ha fatto scoprire la profonda relazione tra le due discipline e la grande valenza educativa di un lavoro unificante per favorire l'acquisizione di competenze matematiche, linguistiche e trasversali. Da questo punto di vista, nel documento Indicazioni Nazionali nuovi scenari (Ministero dell'Istruzione, dell'Università e della ricerca [MIUR], 2018) si legge:

\begin{abstract}
«La matematica [...] permette anche di sviluppare competenze trasversali importanti attraverso attività che valorizzano i processi tipici della disciplina: "In particolare, la matematica [...] contribuisce a sviluppare la capacità di comunicare e discutere, di argomentare in modo corretto, di comprendere i punti di vista e le argomentazioni degli altri." Tali competenze sono rilevanti per la formazione di una cittadinanza attiva e consapevole, in cui ogni persona è disponibile all'ascolto attento e critico dell'altro e a un confronto basato sul riferimento ad argomenti pertinenti e rilevanti. In particolare, l'educazione all'argomentazione può costituire un antidoto contro il proliferare d'informazioni false o incontrollate».
\end{abstract}

(MIUR, 2018, p. 12)

Comunicare, discutere, argomentare, comprendere le argomentazioni degli altri, sono competenze che evidenziano il legame tra le due discipline e rendono il lavoro "italmatico" fondamentale per la formazione di cittadini attivi e consapevoli, dotati di senso critico, capaci di avere un dialogo costruttivo con gli altri.

\section{Il percorso}

Nel percorso di riflessione e sperimentazione intrapreso ci siamo concentrati prevalentemente sulle seguenti tre tematiche:

- la comprensione del testo e il ruolo del linguaggio nella risoluzione dei problemi (par. 3.1);

- le storie e le narrazioni in matematica (par. 3.2);

- la matematica e la ludolinguistica (par. 3.3).

Un'attenzione particolare è stata riservata al lessico, alla comunicazione e all'argomentazione, aspetti trasversali a tutti gli ambiti di contenuto. 
Alcune esperienze realizzate nel nostro primo anno di lavoro sono state esposte e presentate nella mostra/laboratorio "Intrecci tra matematica e italiano: storie, giochi e sfide" realizzata in occasione del festival Matematicando edizione 2018, ${ }^{3}$ ma la nostra attività sta ancora proseguendo e, ormai, non possiamo più prescindere dall'intreccio che lega queste due discipline.

\subsection{La comprensione del testo e il ruolo del linguaggio nella risoluzione dei problemi}

Generalmente, a scuola, un problema viene proposto agli allievi attraverso un testo orale o scritto, dove la componente linguistica risulta determinante per la risoluzione; parole sconosciute, elementi linguistici che non fanno parte dell'esperienza degli alunni, mancanza di conoscenza enciclopedica e struttura del testo complessa: sono solo alcuni degli aspetti che possono compromettere la comprensione delle situazioni ancora prima di arrivare alla trasformazione del testo in linguaggio matematico e alle successive fasi di risoluzione e interpretazione dei risultati. È dunque importante che gli alunni sviluppino buone competenze di lettura e di comprensione profonda dei testi e delle informazioni.

Guidati dalle due docenti, come gruppo abbiamo approfondito l'importanza di rendere consapevoli gli alunni delle finalità e delle diverse strategie di lettura, a seconda delle tipologie testuali, attraverso attività esplicite e specifiche per il raggiungimento di questa competenza (Demartini \& Sbaragli, 2019). In collaborazione con gli insegnanti dell'area linguistica, abbiamo sviluppato percorsi di approfondimento sulle tecniche di lettura per le diverse classi.

Diverse strategie di lettura. Agli alunni di alcune classi terze e quarte primaria, ad esempio, è stata proposta l'attività "Che lettore sei?".

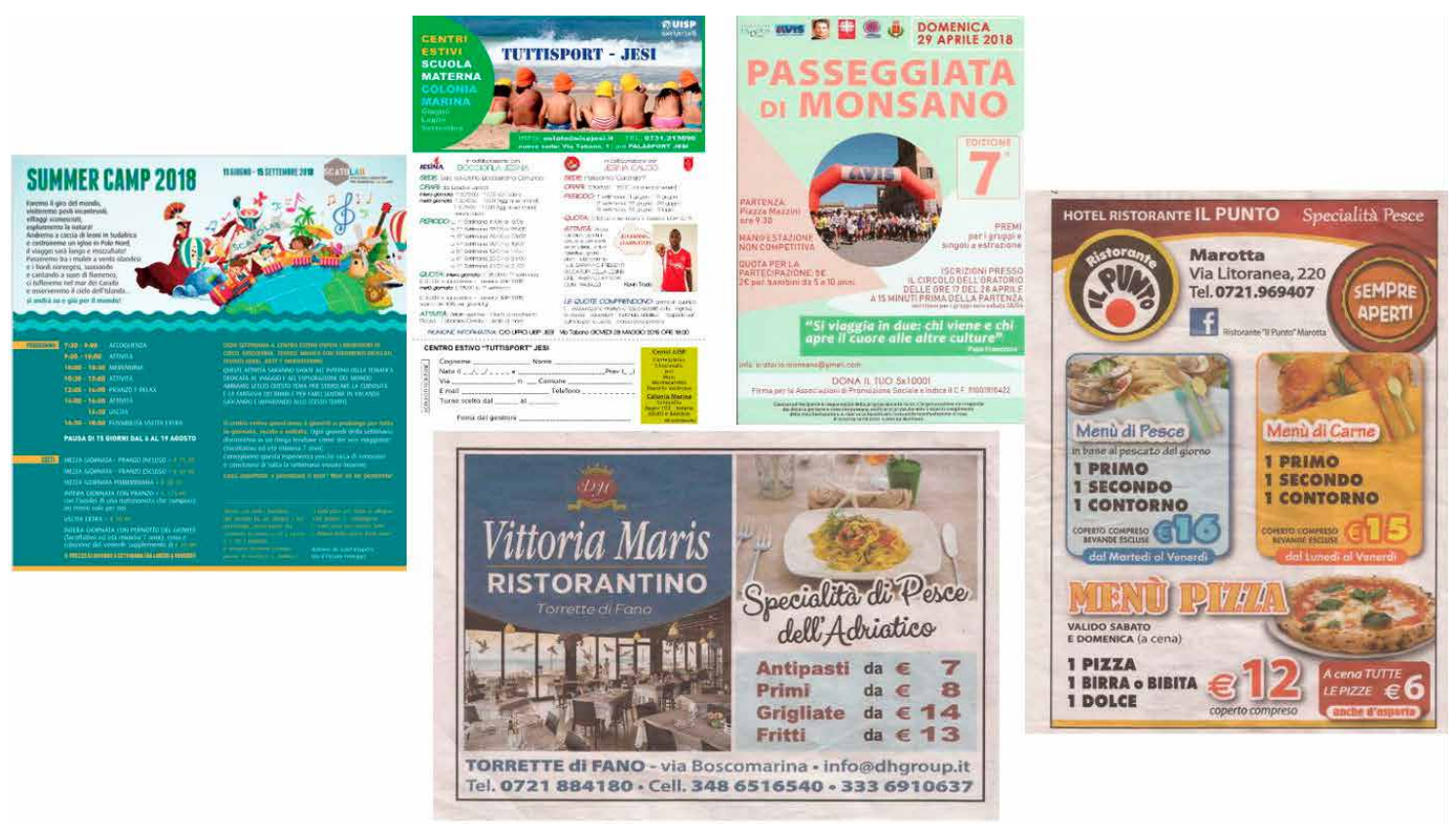

Figura 1. Volantini usati per l'attività "Che lettore sei?".

Sono stati presentati cinque volantini (Figura 1) di altrettanti ristoranti e una scheda con una serie di domande in cui si chiedeva di compiere riflessioni, via via sempre più specifiche, per far sì che la famiglia Rossi riuscisse a soddisfare le diverse richieste riportate nella scheda stessa. (vedi Allegato 1). Completato il lavoro individualmente, si è passati alla riflessione collettiva durante la quale i ragazzi

3. La scheda didattica è consultabile al seguente link https://www.matematicando.supsi.ch/risorse-didattiche/intrecci-tramatematica-e-italiano/. 
hanno riconosciuto due tipologie di lettura: quella "a balzi" in cui l'occhio va velocemente da un'informazione a un'altra, scegliendo quelle importanti, e quella lenta che risulta più intensiva e nella quale ci si sofferma a ripercorrere determinati passi del testo.

Successivamente è stata proposta la lettura della fiaba "Il principe e l'orco" e, in questo caso, gli alunni hanno affermato di aver letto tutto il testo "riga per riga abbastanza velocemente".

Alla fine, gli allievi hanno scelto alcuni animali per rappresentare le diverse tipologie di lettura (Figura 2): la lettura a balzi (lettura esplorativa) è stata associata alla rana, quella veloce al leopardo (lettura estensiva) e quella lenta alla tartaruga (lettura intensiva).

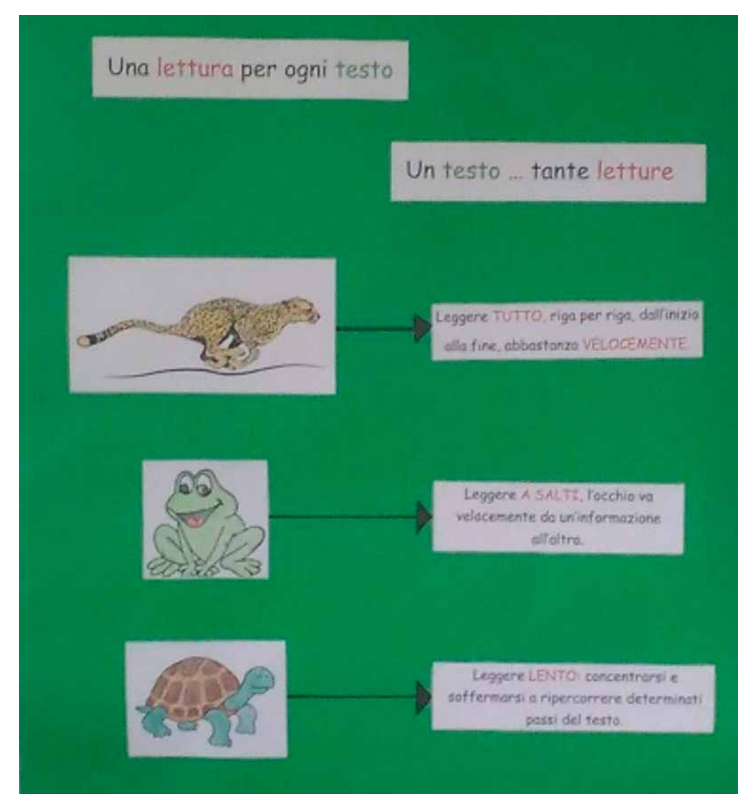

Figura 2. Rappresentazione dei tipi di lettura.

In tutte le classi, dalla seconda primaria, alla terza secondaria di primo grado, abbiamo svolto il lavoro sui diversi tipi di lettura a seconda del contesto, delle finalità che si vogliono raggiungere e del tempo a disposizione, verificando l'utilità di questo approfondimento e l'importanza dell'acquisizione della consapevolezza, da parte degli alunni, che non tutti i testi si leggono allo stesso modo e che lo stesso testo deve essere letto più volte e in modi differenti per raggiungere una comprensione profonda.

Con il nostro percorso di formazione, abbiamo compreso che non sempre le strategie adottate per facilitare la comprensione erano efficaci per guidare gli alunni nell'esplorazione del testo, nell'interrogare i contenuti e nello sviluppare strategie utili alla comprensione profonda delle situazioni, sia dal lato linguistico sia matematico.

Porsi domande per comprendere il testo. Ci siamo resi conto che le nostre richieste più comuni "Leggi bene", "Hai letto con attenzione?", "Rileggi il testo e concentrati", ... avevano una scarsa efficacia perché troppo generiche. Dopo gli approfondimenti teorici suggeriti dalle nostre esperte, abbiamo capito che era necessario fornire indicazioni più precise, specifiche, e abbiamo lavorato sulla riformulazione delle domande guida per la comprensione, individuando molte volte insieme agli alunni le domande utili per soffermarsi sui punti critici, allo scopo di facilitare la comprensione.

Con il problema "Finestre illuminate" (Figura 3) (tratto da Rally Matematico Transalpino maggio-giugno 2014) presentato in diverse classi quarte e quinte primarie abbiamo voluto indirizzare l'attenzione dei bambini e guidare la comprensione del testo con domande stimolo del tipo: La situazione ti è chiara?, Quali sono le parole o le frasi che ti sembrano difficili?, Ti viene chiesto di fare qualcosa?, Che tipo di testo hai letto? ecc. 


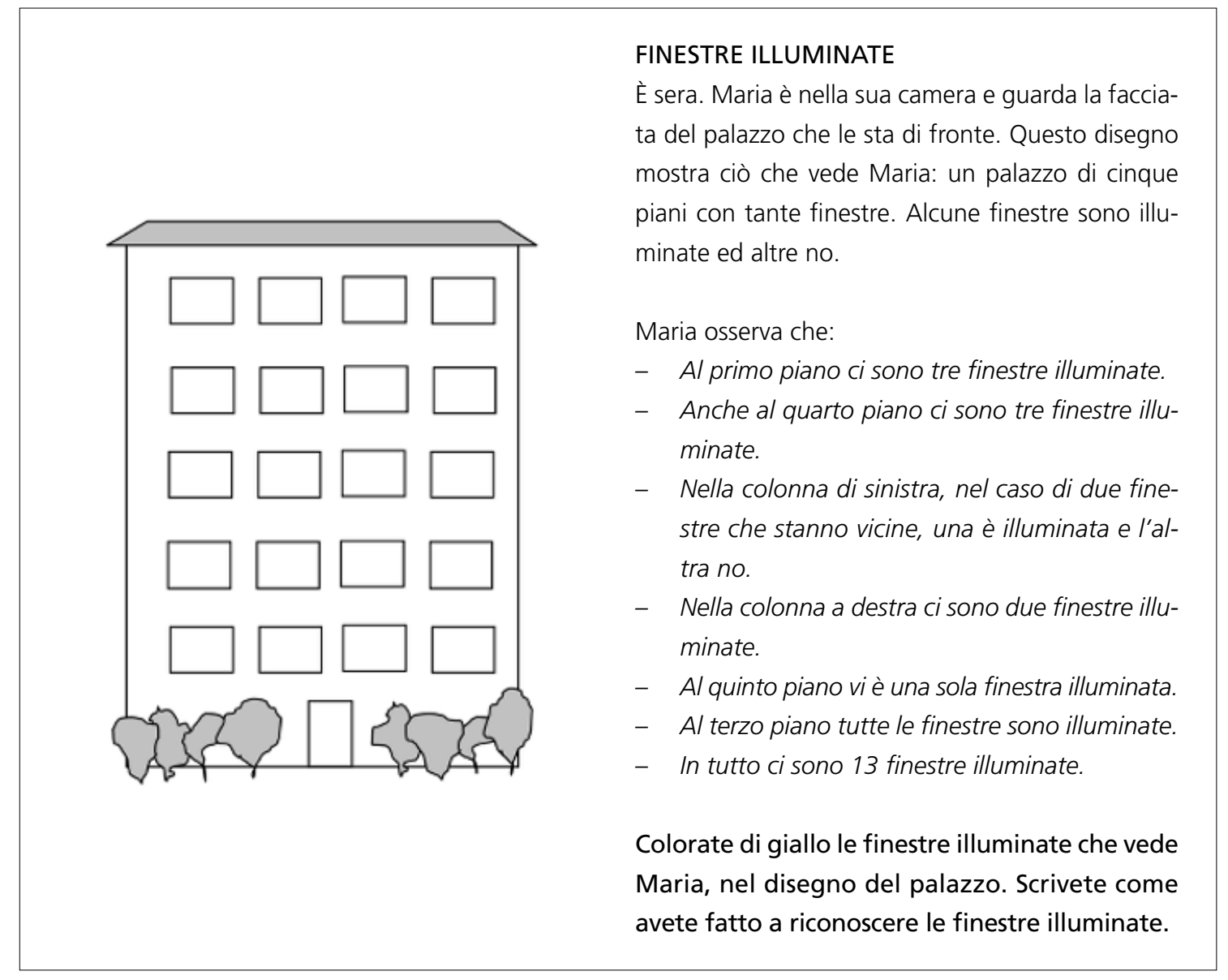

Figura 3. Problema "Finestre illuminate".

Partendo dall'analisi del testo, abbiamo riflettuto sulle sue caratteristiche e sul lessico utilizzato. In generale si è osservato che in questo testo non sono presenti valori numerici e la complessità sta nella concatenazione con implicazioni logiche delle indicazioni fornite; per riuscire a ordinare le informazioni in maniera efficace, risolvendo il problema, sono necessarie una lettura attenta e una comprensione profonda.

Dalla sperimentazione effettuata in diverse classi è emerso che gli alunni a cui sono state rivolte le domande che indirizzavano l'attenzione sui punti critici hanno avuto meno difficoltà nella risoluzione, rispetto ad altri allievi di classi analoghe che hanno ricevuto il testo senza il supporto delle domande guida.

Basandoci sulla consapevolezza acquisita che le capacità di fare ipotesi, trarre inferenze, fare collegamenti e compiere deduzioni, sono aspetti cruciali per la comprensione, abbiamo provato a "spezzettare" testi di problemi per abituare gli alunni ad anticipare elementi semantici, lessicali, grammaticali, a fare ipotesi su un testo di un problema ancora prima di conoscere la richiesta, a sviluppare strategie per organizzare in modo logico gli elementi di un testo, a memorizzare e trattenere le informazioni semantiche necessarie senza farsi condizionare da elementi fuorvianti.

Un esempio è dato dall'attività sperimentata sul problema "Cioccolatini troppo buoni" (Rally Matematico Transalpino, maggio-giugno 2006) proposto ad alunni dalla terza primaria in poi e, in maniera semplificata anche in seconda. 


\section{Cioccolatini troppo buoni}

I cioccolatini di questa scatola erano disposti in modo regolare quando era piena:

- nella prima riga, due cioccolatini tondi al latte erano seguiti da un cremino quadrato al cioccolato fondente, poi da due tondi al latte, poi da un cremino, poi da due tondi al latte, ..

- la seconda riga cominciava con un cremino seguito da due tondi di latte, poi da un cremino, ...

- la terza riga era come la prima, la quarta come la seconda e così via.

Alcuni cioccolatini sono già stati mangiati e ne restano solo 28.

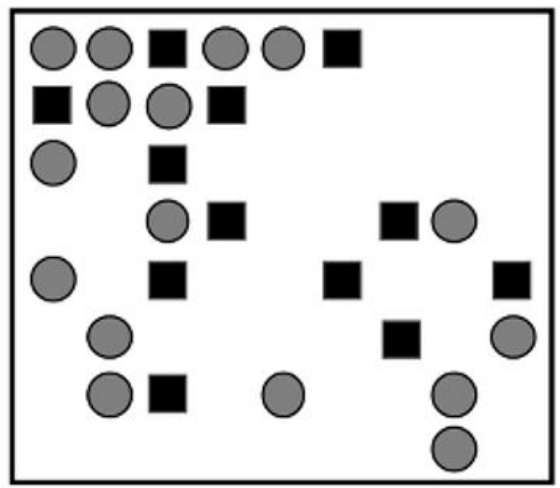

Quanti cioccolatini tondi al latte sono già stati mangiati?

E quanti cremini?

Spiegate come li avete contati

Figura 4. Problema "Cioccolatini troppo buoni"

In Figura 4 il testo è completo, ma agli alunni è stato proposto presentando ogni elemento in successione, sfidandoli a continue anticipazioni, inventive e scoperte: prima abbiamo mostrato soltanto l'immagine chiedendo Che cosa rappresenta secondo te questa immagine?, Di cosa parlerà il problema?; poi all'immagine abbiamo aggiunto il titolo, continuando a stimolare la formulazione di ipotesi Di che cosa si parlerà?, Che cosa rappresenta questa immagine?, Che cosa chiederà il problema?, Sarà adatto per la nostra classe?; quindi abbiamo aggiunto l'inizio del testo, sollecitando anche la riflessione sul lessico Cosa vuol dire "disposti in modo regolare"? ecc. e, in seguito, sono state presentate tutte le informazioni che completano il testo.

L'attività ha molto coinvolto gli alunni, i quali hanno attivato confronti serrati, incuriositi e stimolati dalle informazioni che, arrivando in successione, andavano a confermare o a smentire le ipotesi precedenti. Al termine del lavoro orale, suddivisi in coppie, i ragazzi hanno risolto il problema e verbalizzato il ragionamento. Tutti, anche gli alunni che in genere hanno maggiori difficoltà, hanno trovato questa attività stimolante e utile per facilitare la comprensione, per organizzare in modo logico gli elementi del testo e memorizzare e trattenere le informazioni semantiche necessarie (si veda l'esperienza in una classe terza primaria in Allegato 2).

L'importanza della varietà nei problemi. Abbiamo cercato di proporre testi di problemi che non presentassero i classici stereotipi, scegliendo testi interessanti, che a volte abbiamo modificato a seconda delle esigenze, dalla banca dati delle prove Invalsi (https://www. gestinv.it/Index.aspx) dall'archivio delle prove del Rally Matematico Transalpino (https://armtint.eu/), dai Giochi Matematici del Mediterraneo (https://www.accademiamatematica.it/esercitiamoci) e molti ne abbiamo inventati e costruiti noi stessi.

Abbiamo imparato ad analizzare, discutere e rendere esplicite le motivazioni alla base della scelta di un problema da proporre agli alunni e a esplicitare anche le nostre aspettative prima di proporre un testo in classe, per poi confrontarle con quello che era effettivamente avvenuto dopo aver posto il problema agli alunni e aver indagato sui loro processi risolutivi. 
Prendendo spunto dall'analisi di alcuni quesiti Invalsi ${ }^{4}$, prima di proporli agli alunni abbiamo analizzato i testi dei problemi in base all'ambito di riferimento, allo scopo, al processo che avrebbero sollecitato, ai traguardi e agli obiettivi. Abbiamo cercato di prevedere la riuscita degli alunni, le difficoltà che avrebbero potuto incontrare, e abbiamo cercato di ipotizzare le strategie risolutive che avrebbero potuto mettere in atto. Si sono verificate situazioni molto interessanti che a volte hanno confermato - altre volte smentito - le nostre previsioni.

Il seguente problema, tratto dalle prove Invalsi della prima secondaria di primo grado a.s. 2003/2004 (Figura 5) è stato rielaborato da noi nel seguente modo: Alberto ha 27 figurine, Giovanni ne ha meno di Alberto ma più di Giorgio che ne ha 19. Quante figurine ha Giovanni? Questa riformulazione ci consentiva di lasciare la risposta aperta, cosa che inizialmente ci sembrava non dovesse creare troppi problemi, ma non è andata così.

27. Alberto ha 27 figurine; Giovanni ne ha meno di Alberto ma più di Giorgio che ne ha 19. Quale delle seguenti affermazioni è sicuramente FALSA? Giovanni ha...
A. 25 figurine
B. 22 figurine.
C. 20 figurine.
D. 18 figurine.

Figura 5. Quesito 27 - Prove Invalsi 2003/2004 - Prima secondaria di primo grado.

Il problema, nella sua brevità, presenta delle particolarità: pur essendo presenti nel testo dei valori numerici, non si risolve con dei calcoli aritmetici; gli alunni avrebbero dovuto esprimere la risposta come intervallo di numeri compresi tra 19 e 27, che i più grandi potevano anche rappresentare con una appropriata simbologia numerica.

Il problema è stato proposto in diverse classi quarte e quinte primaria e prima secondaria di primo grado; nella metà di queste classi non sono state poste domande volte a stimolare una comprensione; nell'altra metà sono state poste domande di comprensione del testo del tipo: Hai compreso il testo?, Ci sono frasi poco chiare?, Comprendi la richiesta?, Pensi che un disegno ti possa aiutare nella soluzione?.

In entrambi i casi si sono lasciati gli allievi liberi di risolvere il problema usando qualsiasi tipo di rappresentazione: schema, tabella, disegno ecc. Dove sono state poste le domande aggiuntive di comprensione del testo i risultati sono stati più soddisfacenti, ma in tutte le classi sono emerse diverse difficoltà. A conclusione del lavoro, in tutte le classi si sono confrontati i procedimenti risolutivi chiedendo a ciascuno di motivare le proprie scelte e di condividere le difficoltà incontrate: alcuni avevano usato la rappresentazione grafica, senza giungere a una soluzione, altri avevano eseguito delle operazioni, alcuni avevano rinunciato a risolverlo (Figura 6); una minima parte degli alunni aveva scritto che non si poteva conoscere esattamente il numero delle figurine di Giovanni, ma che si poteva dire soltanto che era un numero compreso tra 20 e 26 (Figura 7).

La discussione in classe è stata molto utile per riflettere sulle difficoltà determinate dal condizionamento dei numeri presenti nel testo che avevano portato gli alunni alla ricerca di un'operazione riso-

4. Alcune di queste analisi si trovano al sito https://invalsi-areaprove.cineca.it/index.php?get=static\&pag=qdr. 
lutiva e di una soluzione esatta e sul disagio causato dal fatto che nessuna delle operazioni conosciute sembrava andar bene: non ho capito nulla, non so come andare avanti...
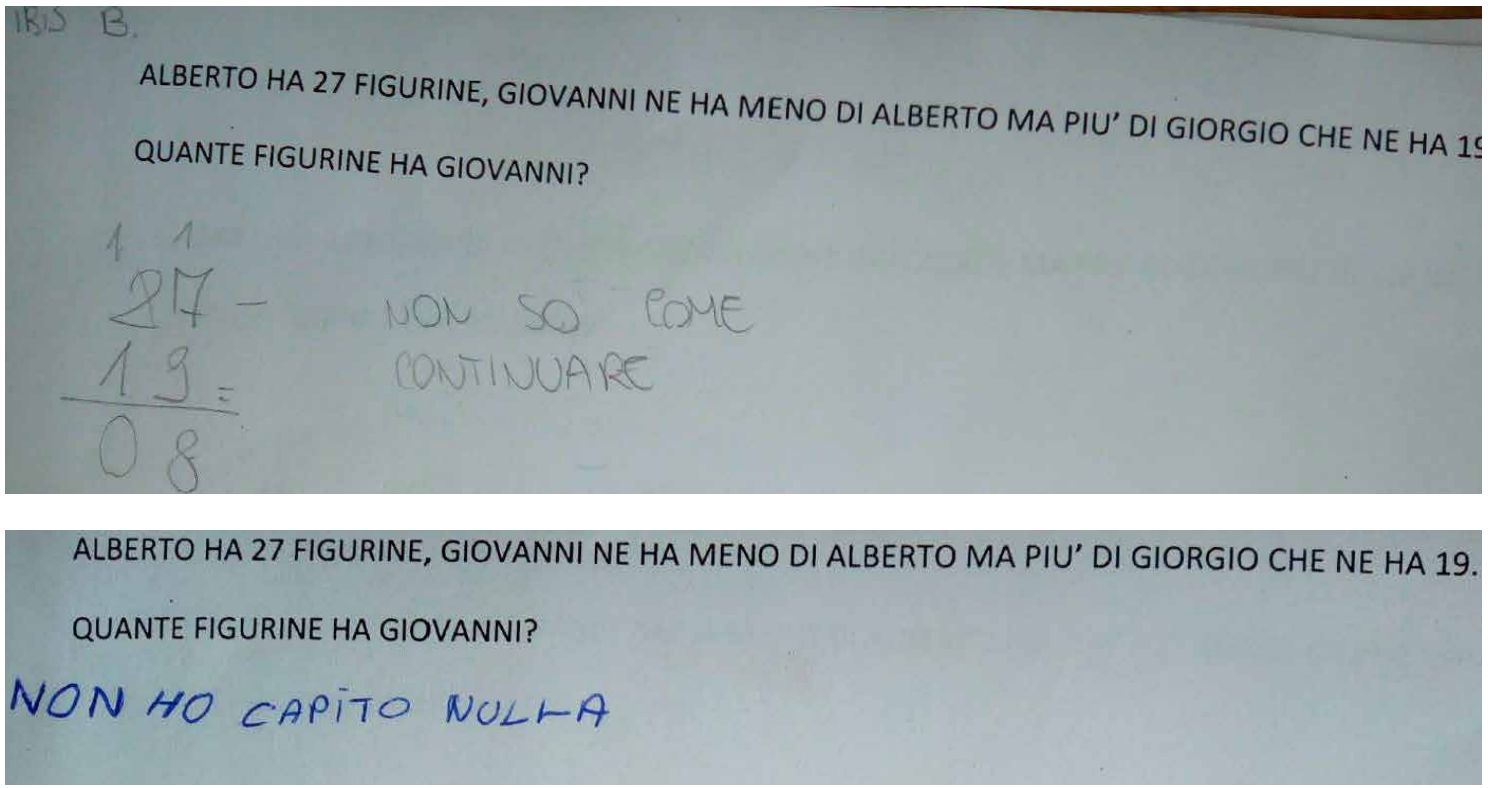

Figura 6. Esempi di soluzioni erronee del problema "Le figurine di Giovanni".
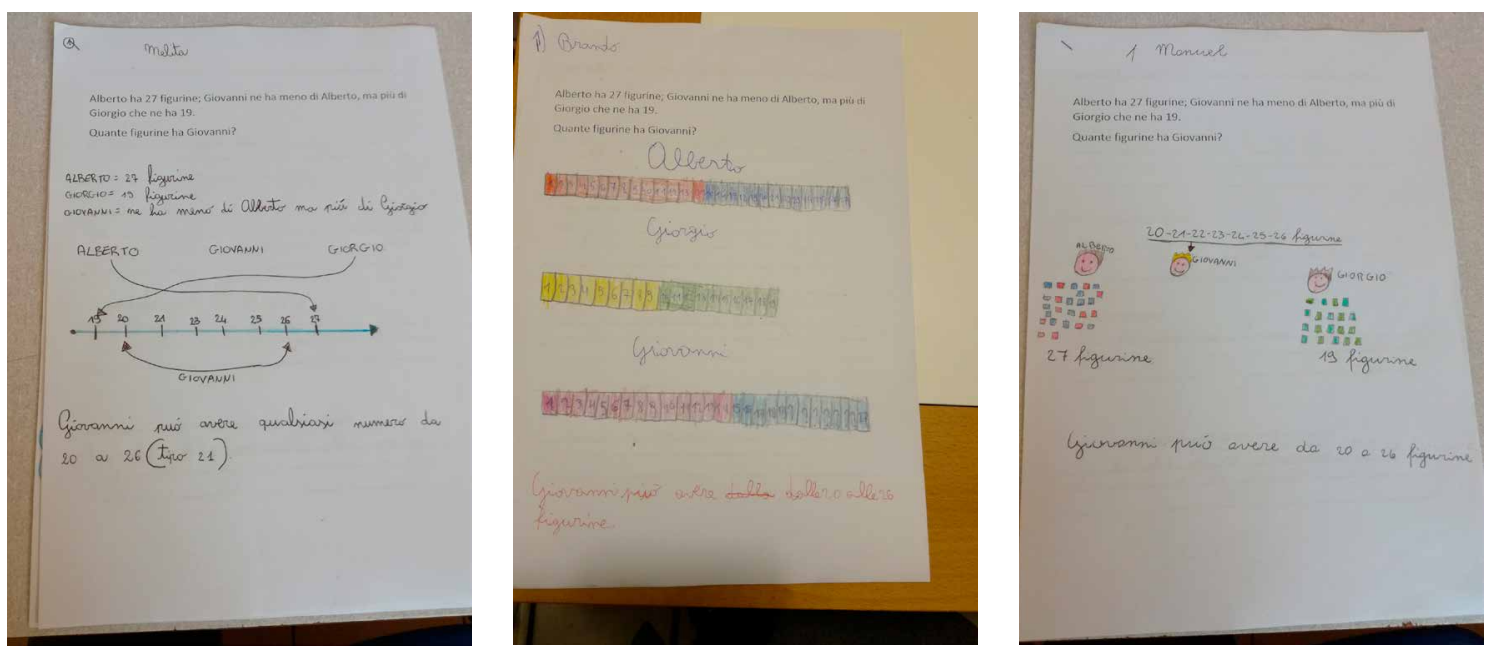

Figura 7. Esempi di soluzioni figurali del problema "Le figurine di Giovanni".

La condivisione dell'esperienza nel gruppo ha rinforzato la nostra convinzione che è assolutamente necessario variare le tipologie di problemi, le metodologie adottate e soprattutto lasciare libertà nella scelta delle strategie risolutive e delle relative rappresentazioni. Abbiamo riflettuto molto sulle diverse tipologie di problemi (senza numeri, con più soluzioni, problemi bucati...), usato e inventato testi di vario genere (partendo da racconti, volantini, fumetti, regole di giochi, situazioni reali...), variando anche le modalità didattiche e le richieste e accettando risposte e soluzioni presentate in modo personale e creativo.

Il problema "Scoiattoli nel bosco" (Figura 8), ad esempio, proposto ad alunni di una prima primaria, si presta ad essere risolto in modi diversi. 


\section{Oggi la maestra Pina porta la sua classe di 19 bambini in palestra per giocare agli "SCOIAT- TOLI NEL BOSCO".}

Immaginate di essere degli scoiattoli che vagano nel bosco in cerca di ghiande per le provviste invernali.

I cerchi sul pavimento sono gli alberi dentro i quali potrete rifugiarvi in caso dell'arrivo del temporale.

Quando batterò le mani vorrà dire che il temporale è in arrivo: dovrete correre tutti al riparo nei cerchi, nessuno scoiattolo dovrà rimanere senza rifugio!

Ma attenti: in ogni cerchio possono ripararsi al massimo 2 scoiattoli!

- Alla classe basteranno 9 cerchi per giocare a questo gioco?

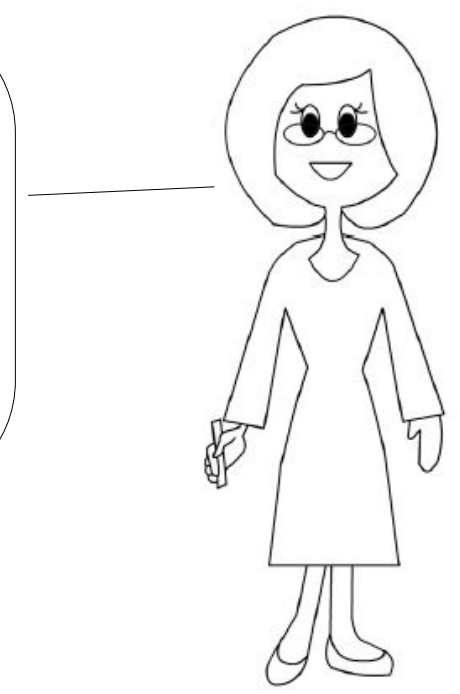

Si No

- Spiegate perché.

Figura 8. Problema "Scoiattoli nel bosco".

Dopo una prima lettura del testo (adattato al numero di alunni presenti in ogni classe) fatta dagli insegnanti, i bambini hanno letto individualmente il problema. Per verificarne la comprensione abbiamo utilizzato una conversazione collettiva, durante la quale gli alunni hanno iniziato ad avanzare alcune ipotesi di risposta. Suddivisi in coppia, poi, si sono confrontati e hanno usato le strategie che ritenevano più opportune per risolvere il problema. I bambini, privi di troppi condizionamenti essendo più piccoli, hanno scelto strategie e modalità di rappresentazione molto differenti e personali che andavano dalla forma pittorica (Figura 9), alla forma verbale (Figura 10), a quella simbolica (Figura 11).
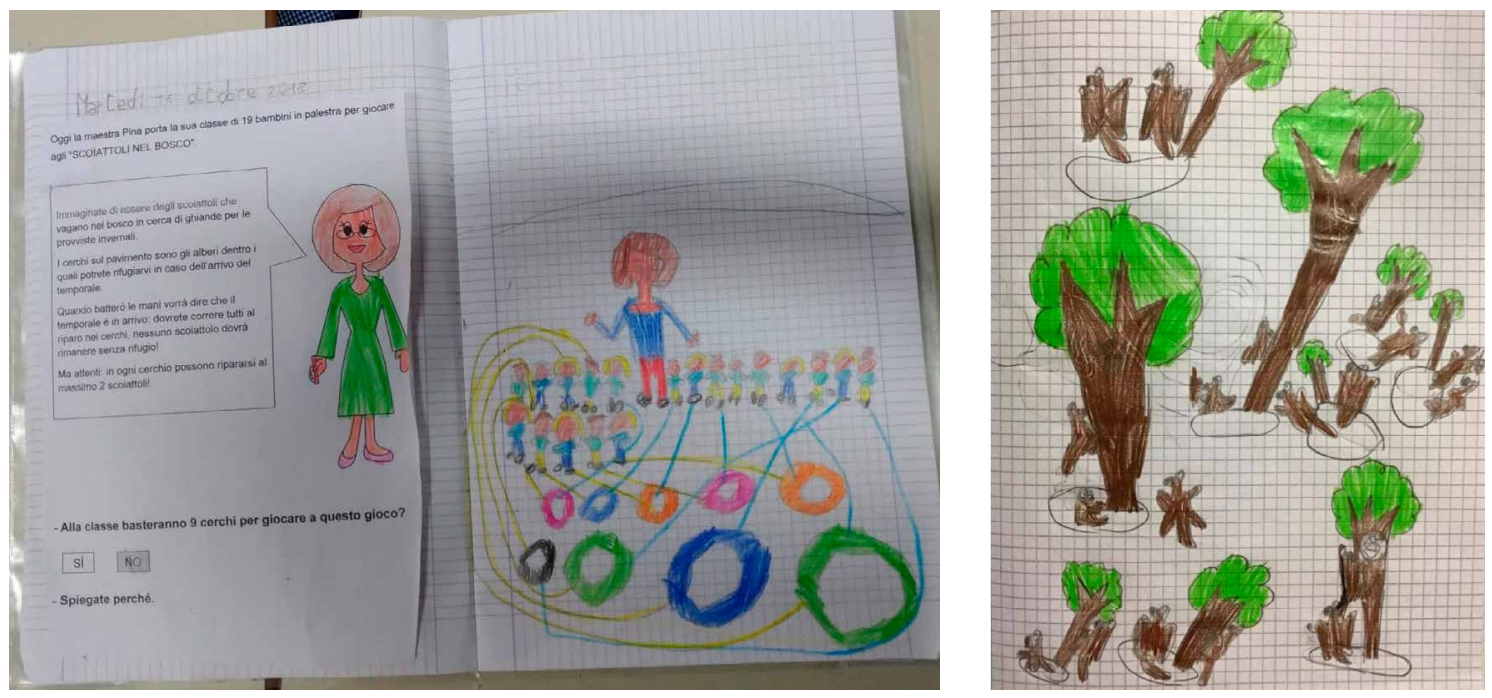

Figura 9. Risoluzioni pittoriche del problema "Scoiattoli nel bosco". 


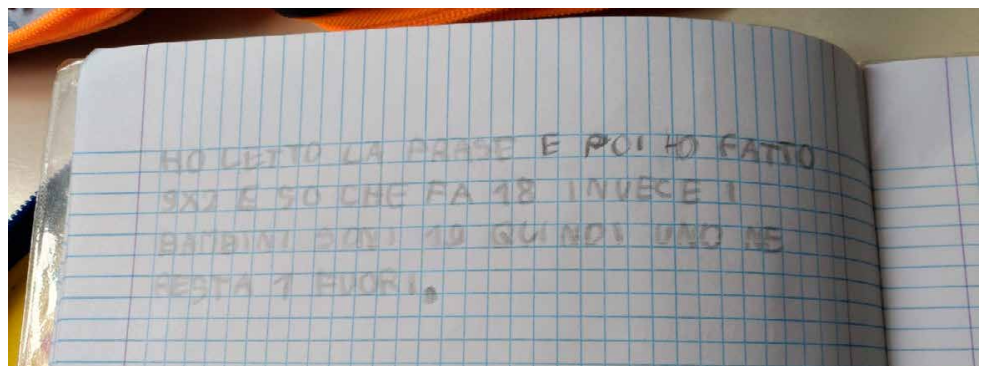

Figura 10. Risoluzione verbale del problema "Scoiattoli nel bosco".
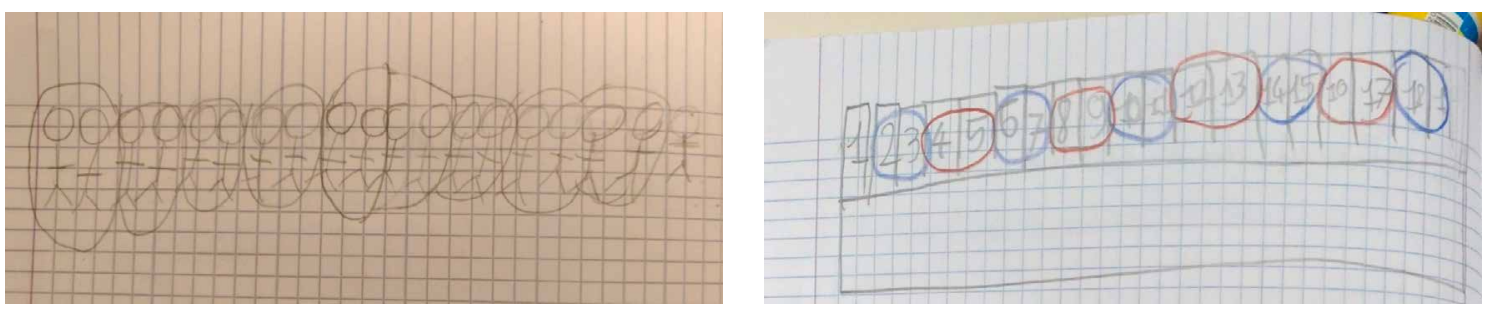

Figura 11. Risoluzioni simboliche del problema "Scoiattoli nel bosco"

Le varie proposte risolutive prodotte dai bambini cambiavano anche in base al coinvolgimento emotivo derivante dalla narrazione, dallo stile personale, dalle preferenze artistiche o meno, ma tutte sono risultate efficaci. Con questi lavori di riflessione ci siamo convinti sempre più che questa creatività è preziosa e che non dobbiamo pretendere soluzioni standardizzate: meglio farci stupire dai nostri alunni lasciando loro la libertà di scegliere processi risolutivi e rappresentazioni varie.

Per noi è risultato inoltre importante coinvolgere attivamente gli alunni facendo loro inventare o riformulare dei testi, delle domande, delle strategie risolutive, abituandoli alla discussione e al confronto tra pari e con gli adulti. A questo proposito abbiamo sperimentato diverse strategie: riscrittura di testi di problemi; invenzione di testi partendo da immagini, da fumetti o da risoluzioni fornite, oppure prendendo spunto dalla lettura di classici di narrativa, come ad esempio "Pippi Calzelunghe" di A. Lingdren (2010), "Matilde" di R. Dahl (2016), "L'albero del riccio" di A. Gramsci (2019); invenzione di testi con vincoli lessicali, oppure tratti dalle spiegazioni delle regole di giochi, o ancora da situazioni reali o da volantini.

Inventare problemi. Molto interessante ci è sembrata un'attività presentata agli alunni delle classi quinta primaria ai quali è stato chiesto di lavorare su un dépliant di una mostra fotografica che si stava svolgendo in città (Figura 12).

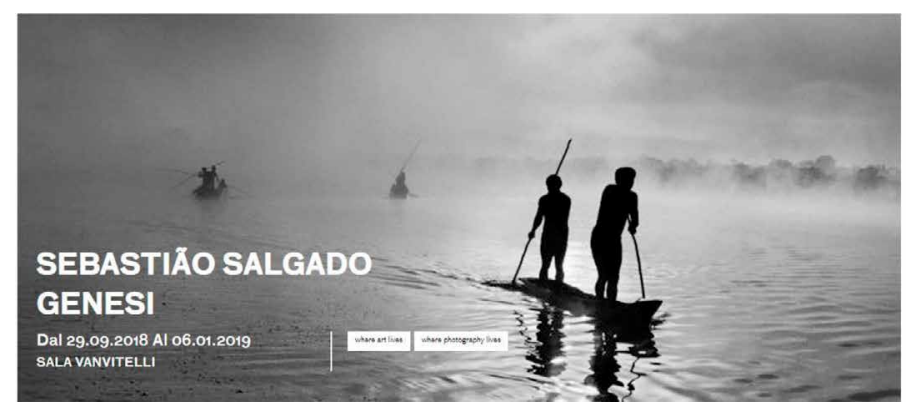




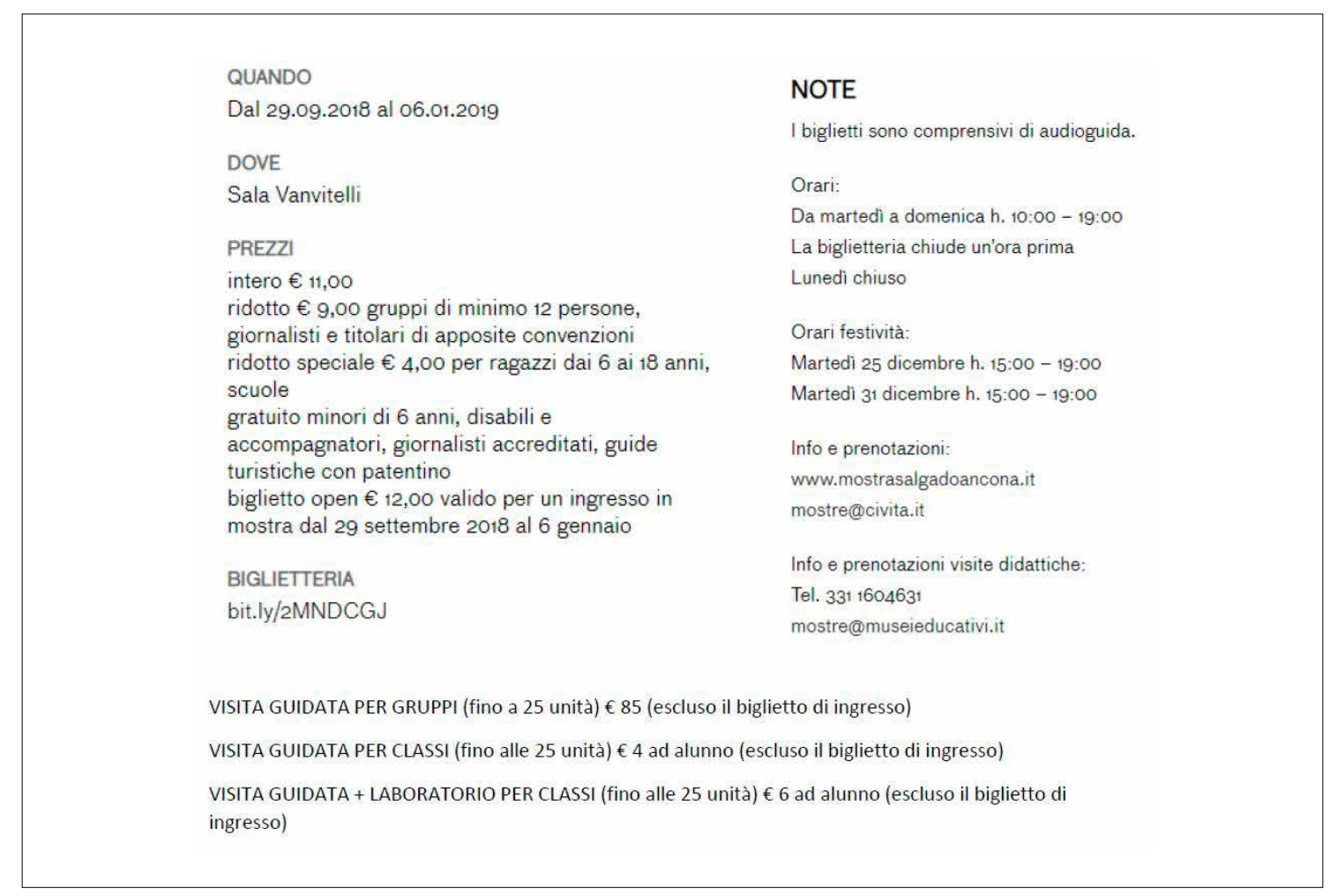

Figura 12. Dépliant mostra fotografica.

Dopo aver consegnato una copia del volantino ai ragazzi suddivisi in coppie, abbiamo chiesto di formulare dieci domande attingendo alle informazioni dal materiale in loro possesso; in seguito abbiamo chiesto di scambiarsi le domande tra le coppie e di rispondere a quelle dei compagni. Infine, abbiamo proposto a ciascuna coppia di controllare le risposte ricevute indietro dai compagni. II confronto collettivo ha evidenziato che ad alcune domande non si poteva rispondere perché erano incomprensibili o mal formulate; molti avevano formulato domande su contenuti strettamente informativi, altri avevano posto domande ipotizzando la visita da parte di famiglie, gruppi, scolaresche composte in vario modo. In diversi casi sono stati necessari chiarimenti, alcuni alunni hanno dovuto riformulare testi e richieste per permettere ai compagni di capire le situazioni presentate e provare a rispondere. Anche i ragazzi generalmente meno coinvolti hanno partecipato attivamente e hanno gradito l'attività perché si lavorava in coppia ed era necessario un confronto sulle scelte da condividere, perché occorreva mettersi nella testa degli altri per scrivere bene le domande in modo che i compagni capissero e perché si doveva affrontare una situazione possibile, realistica; a tal proposito un alunno ha affermato: "potrei andare anche io con la mia famiglia a vedere la mostra e ora so come leggere i biglietti".

Tutte le esperienze che sollecitano il confronto, la motivazione delle proprie osservazioni, il sostegno delle proprie scelte, ci sono risultate preziose per il loro contributo allo sviluppo della capacità di ascolto, di mettersi nei panni degli altri e dello sviluppo di competenze comunicative e argomentative.

Anche il ricorso ad artefatti come i dadi ci ha offerto un notevole appiglio narrativo per inventare storie o problemi, non solo con i più piccoli. Prendendo spunto dai dadi per inventare storie (Rory's story cubes) abbiamo costruito i nostri Math cubes (Figure 13, 14 e 15). Ogni Math cube riguardava un tema specifico: ambiti coinvolti aritmetica o geometria; tipologia di narrazione; ambienti; personaggi; numeri; figure geometriche; risoluzione; tipo di problema. 


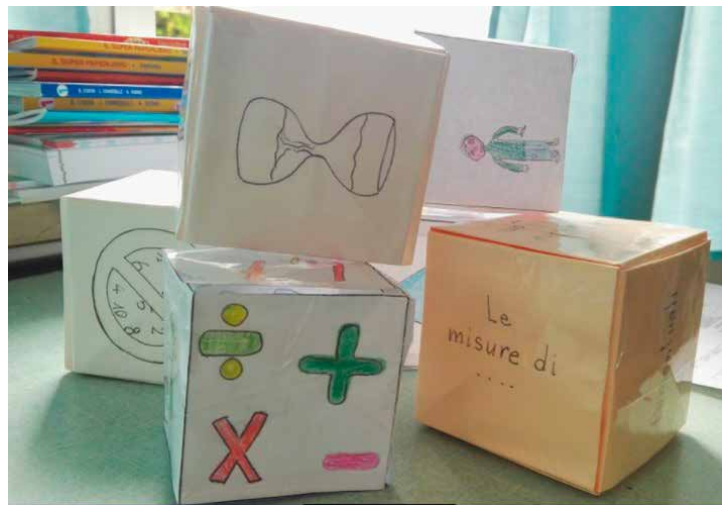

Figura 13. Math cubes.

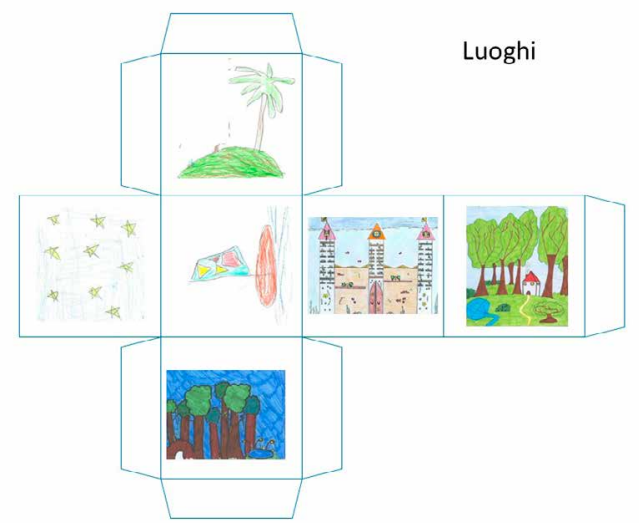

Figura 14. Esempio di sviluppo di Math cubes.

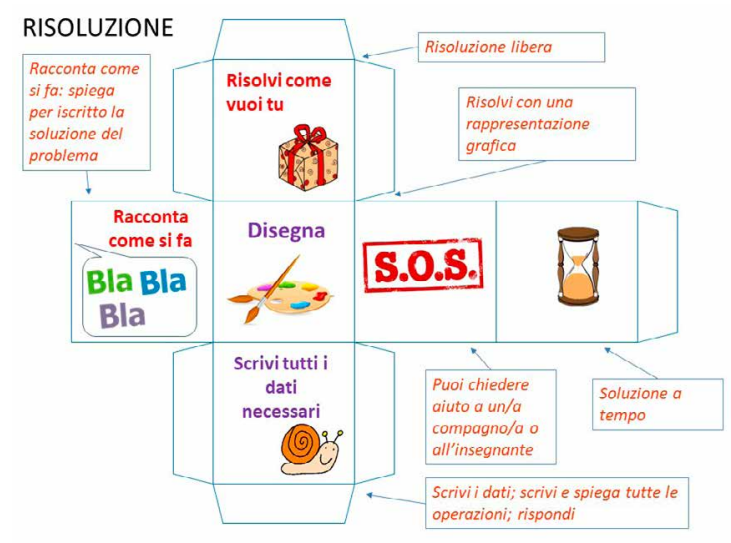

Figura 15. Esempio di sviluppo di Math cubes.

Abbiamo utilizzato i dadi per inventare storie e problemi collegando tutti gli elementi con creatività e fantasia. Le attività che si possono proporre sono molteplici: gli alunni lanciano i dadi poi creano una storia-problema in modo collettivo, usando tutti gli elementi che sono usciti; dividendo la classe in due gruppi, un gruppo lancia i dadi e inizia la storia, I'altro gruppo la completa e poi entrambi i gruppi risolvono i problemi emersi; gli alunni, divisi in squadre si sfidano a suon di problemi, ogni squadra lancia i dadi, inventa il problema e lo passa alle altre squadre.

Sono soltanto alcuni esempi delle tante possibilità offerte dai dadi delle storie, i quali, all'inizio dell'anno scolastico, sono stati utili per attività di accoglienza, per ripassare e consolidare in modo coinvolgente e motivante contenuti matematici e per riflettere sui problemi in modo diverso e divertente. Con lo scambio dei problemi, nel confronto tra pari, non sono mancate osservazioni sulla chiarezza delle informazioni, sull'organizzazione del testo, su come era costruita la storia e sulle parole utilizzate; queste sollecitazioni sono state molto utili per una riflessione attenta sui testi.

Un altro simpatico artefatto che può essere utilizzato per inventare storie e problemi è il classico gioco inferno e paradiso (Figura 16), che è stato usato con i bambini di alcune classi prime per avviare alla scrittura di brevi narrazioni matematiche. 

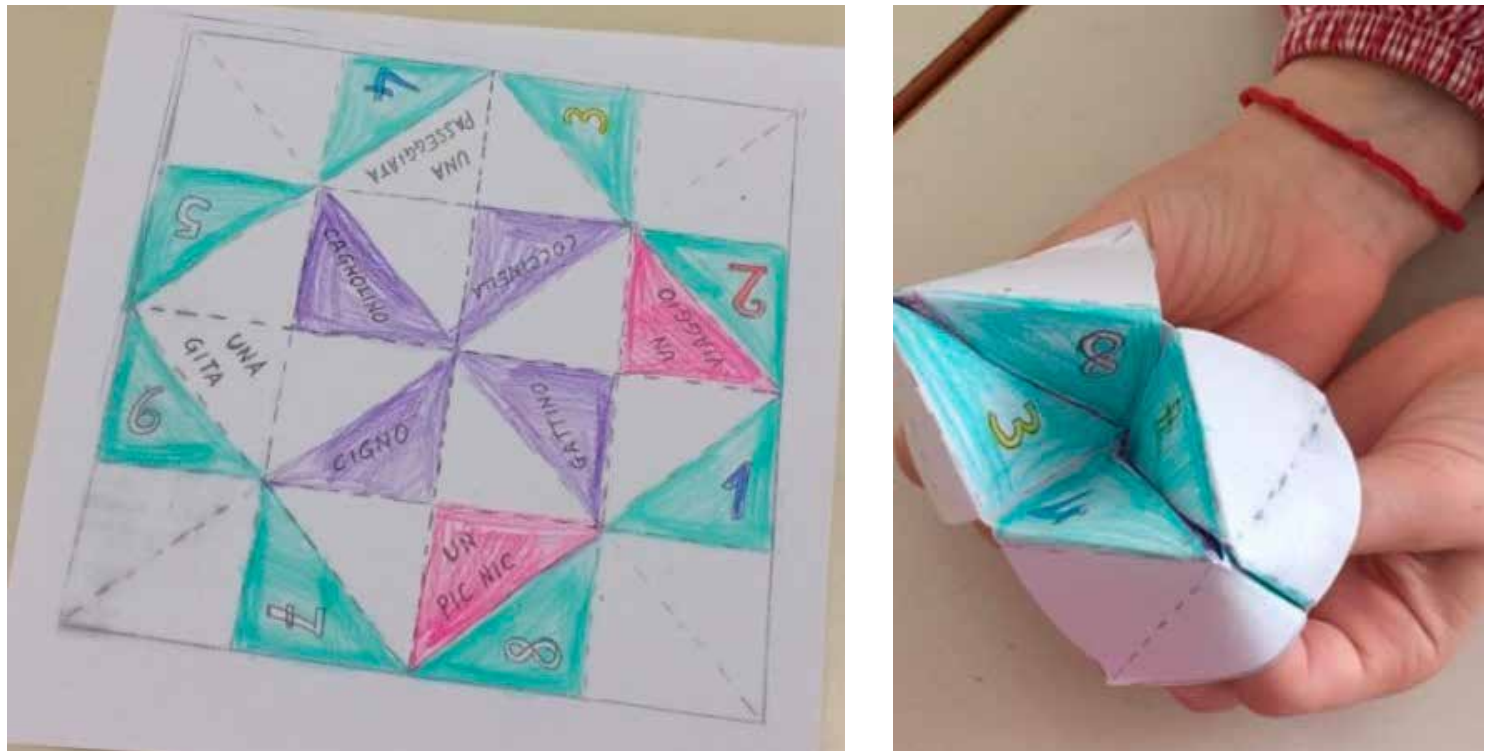

Figura 16. Gioco "inferno e paradiso" per inventare storie e problemi.

Nascosti sotto i numeri dispari ci sono dei nomi di animali (cagnolino, gattino, coccinella, cigno), nascosti sotto i numeri pari ci sono degli eventi (una passeggiata, una gita, un picnic, un viaggio). I bambini hanno costruito questo gioco, poi in coppie, dando spazio alla loro inventiva e fantasia, hanno inventato storie-problema abbinando personaggi e luoghi.

\subsection{Le storie e le narrazioni in matematica}

Nei 20 anni di vita del gruppo di Corinaldo abbiamo sempre utilizzato storie per raccontare la matematica, per introdurre nuovi concetti, per far parlare gli alunni dei concetti appresi, nella convinzione che un racconto, una storia suscita curiosità ed emozioni, conquista l'attenzione degli alunni e favorisce la motivazione all'apprendimento.

Negli ultimi anni I'uso delle storie nella didattica della matematica è divenuto più consapevole, le finalità sono più chiare, pertanto I'applicazione didattica risulta più efficace (Demartini \& Sbaragli, 2015). L'uso delle narrazioni aiuta gli alunni, di tutti i livelli di età, a strutturare il loro pensiero e a dare ordine agli eventi, stimola la curiosità, la creatività e il desiderio di sperimentare e di conoscere.

Storie da inventare (e ascoltare). Ascoltare e inventare storie sviluppa la capacità di interpretare i fenomeni didattici e di osservare/valutare i processi risolutivi, apre la mente ad altri mondi possibili e stimola il lavoro sull'astrazione.

Nella scuola dell'infanzia sono stati realizzati dei bellissimi albi illustrati con storie lette o inventate con i bambini, riguardanti i numeri e le figure. L'Alieno Numerino racconta di un alieno atterrato in uno zoo che si diverte a contare gli animali nelle gabbie. La storia è stata condivisa dagli insegnanti del gruppo, poi ogni scuola ha realizzato l'albo con la tecnica preferita: alcuni gruppi hanno prodotto un albo da sfogliare (Figura 17), altri un albo con le figure mobili per poter giocare. 


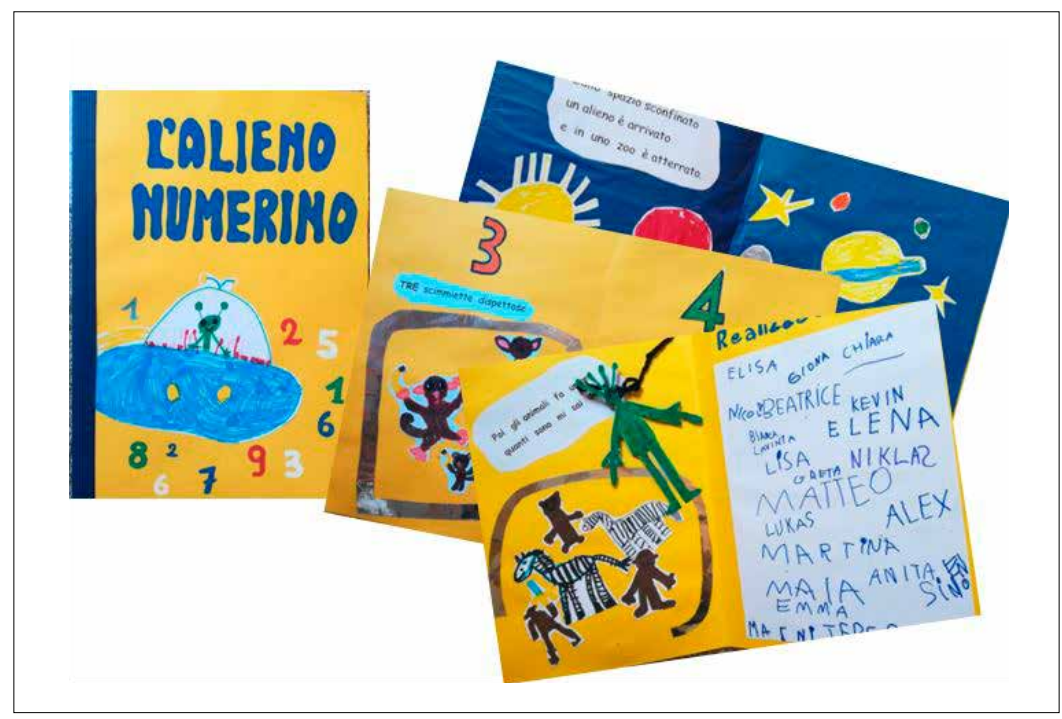

Figura 17. Esempio di albo prodotto dagli allievi partendo dalla storia "Alieno numerino".

Diverse sono le storie che abbiamo inventato nel passato e che spesso ancora utilizziamo per introdurre l'esplorazione delle figure geometriche solide fin dalla scuola dell'infanzia, prassi che è ormai consolidata (Arrigo \& Sbaragli, 2004; Cottino \& Sbaragli, 2005). I bambini manipolano oggetti e li confrontano, scoprono le forme, notano le loro caratteristiche evidenti e le generalizzano. Le loro osservazioni spontanee vengono accolte e convogliate in un percorso organizzato, costituito di occasioni concrete e ragionate, in cui è possibile scoprire, sperimentare e riconoscere in modo giocoso, divertente e coinvolgente le principali figure solide.

Tra queste ci piace ricordare "Il cubo che voleva diventare sfera": una delle prime narrazioni che abbiamo inventato insieme agli allievi per introdurre l'esplorazione delle figure geometriche solide con i bambini dei 5 anni della scuola dell'infanzia e della prima primaria. ${ }^{5}$ Ne siamo particolarmente orgogliosi, perché i marionettisti e attori Cosimo Galiano e Maria Lindeman (Pikku Aasin Nukketeatteri, Vaasa, Finlandia) hanno creato con la nostra storia lo spettacolo di marionette Kuutio \& pallo / Kuben \& bollen, ovvero Cubo e sfera (tecnica: marionette, teatro in nero, attore) da proporre in Norvegia e in Finlandia (Figura 18).

Ogni volta che si guardava allo specchio (il cubo) esclamava: - Come sono brutto! Non sopporto questi vertici in "alto" e in "basso". E questi spigoli... mi danno un aspetto troppo goffo e sgradevole... Un piccolo viaggio tra le differenze per scoprire ed accettare, con gli occhi puri di un bambino, la propria unicità.
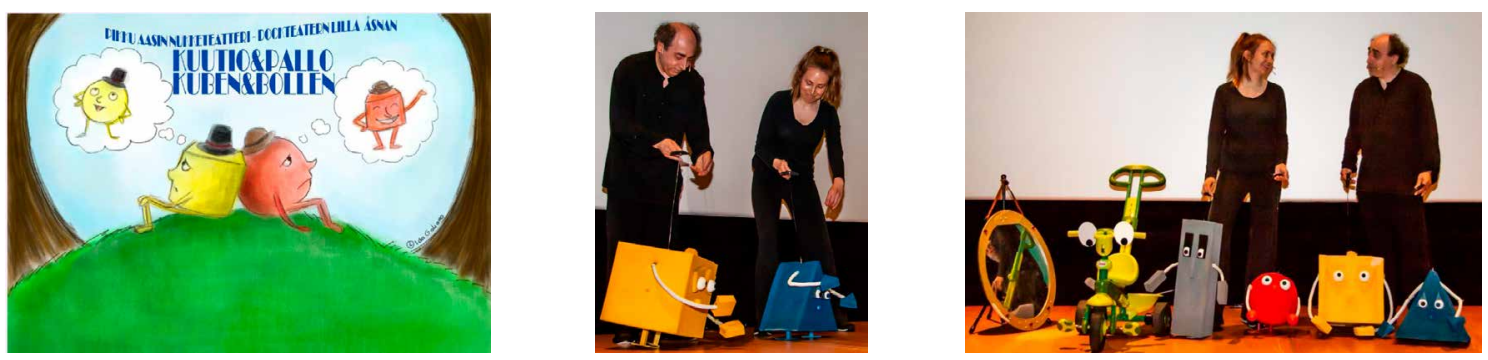

Figura 18. Immagini dello spettacolo di marionette Kuutio \& pallo.

5. Per approfondire la storia si rimanda al link https://rsddm.dm.unibo.it/tipi-rotondi-e-tipi-spigolosi-esperienze-in-3d/. 
Una delle nuove storie alla cui costruzione hanno lavorato gran parte degli insegnanti del gruppo, è "Il Circo Mat-Ita" (Allegato 3, Figure 19 e 20). Si tratta di un racconto finalizzato all'introduzione e alla scoperta di alcune caratteristiche del cubo, del parallelepipedo e della piramide. L'esperienza è stata sperimentata in diverse sezioni/classi, con sviluppi adattati alle diverse situazioni specifiche. In tutti i casi la narrazione si è rivelata molto coinvolgente per gli alunni ed efficace ai fini dell'apprendimento.

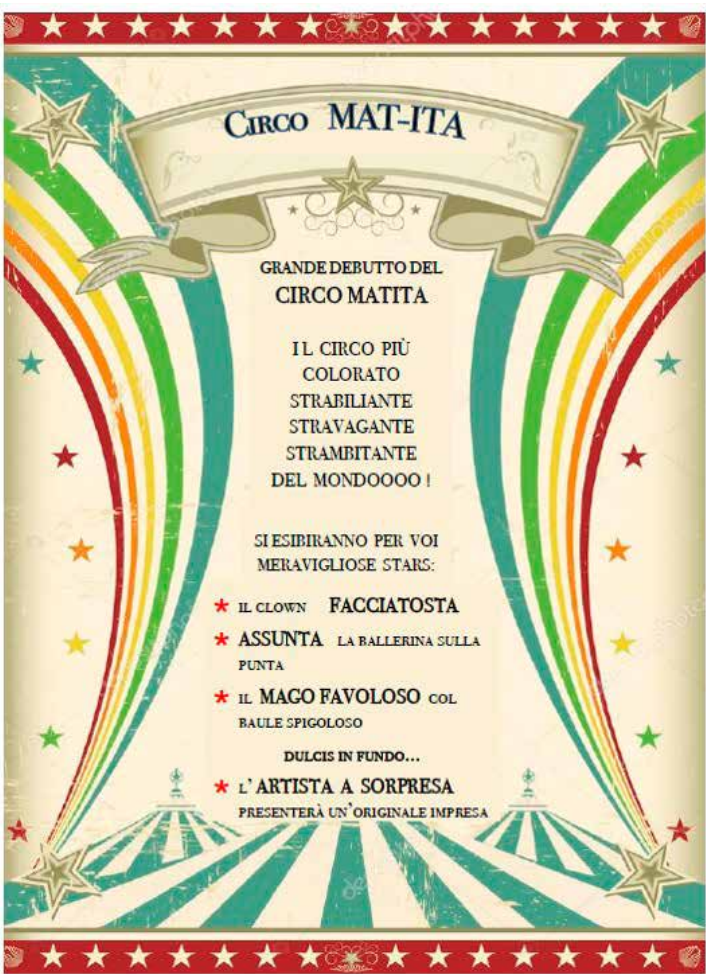

Figura 19. Locandina del circo Mat-Ita.

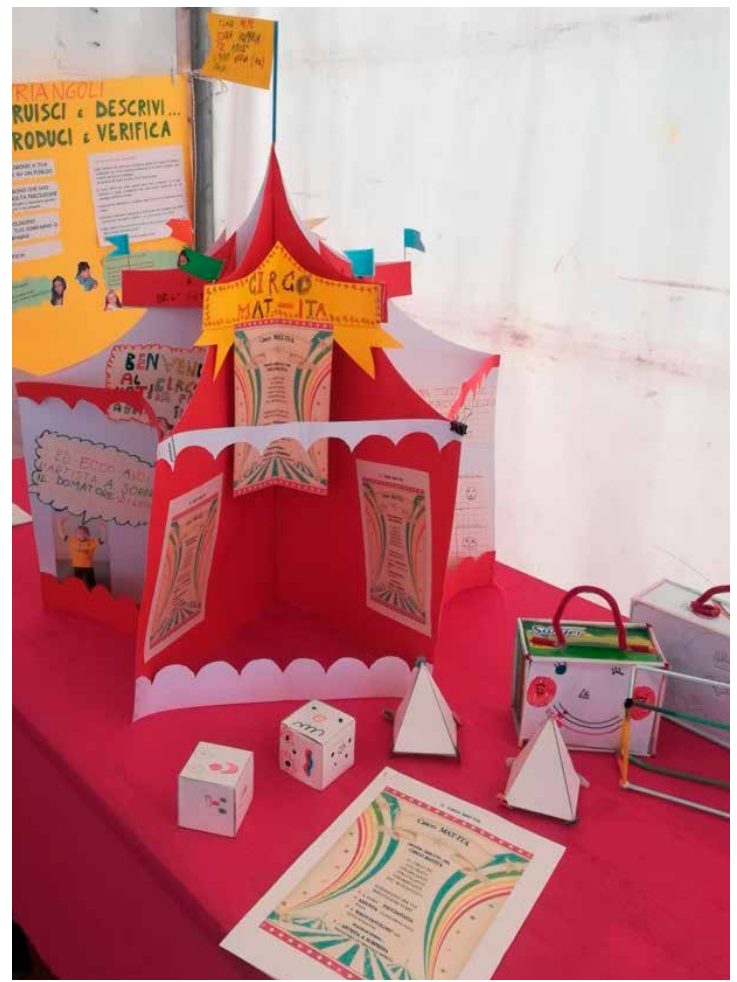

Figura 20. Libro gioco del circo Mat-lta.

Gli alunni della sezione dei cinque anni della scuola dell'infanzia e della classe prima della scuola primaria sono stati catturati dalle vicende degli stravaganti personaggi di questo originale circo e si sono appassionati nello svolgimento dei compiti loro richiesti: disegnare le espressioni sulle facce del clown Facciatosta (Figura 21), preparare le scarpette per Assunta la ballerina sulla punta (Figura 22) e ricostruire il baule del Mago Favoloso (Figura 23). A conclusione della storia, i bambini si sono sbizzarriti nell'invenzione di un artista a sorpresa.

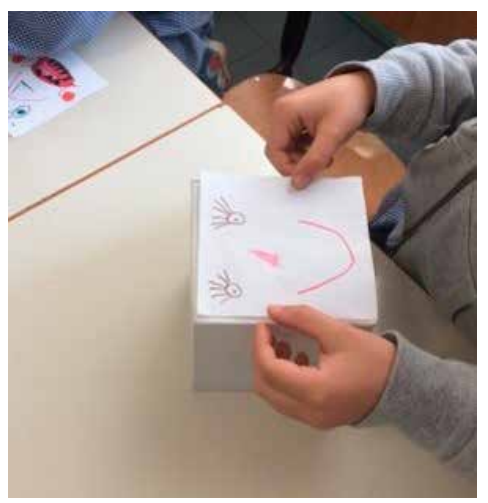

Figura 21. Costruzione del clown Facciatosta.

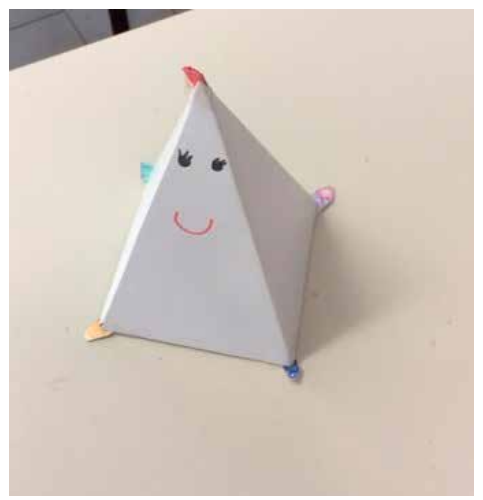

Figura 22. La ballerina Assunta.

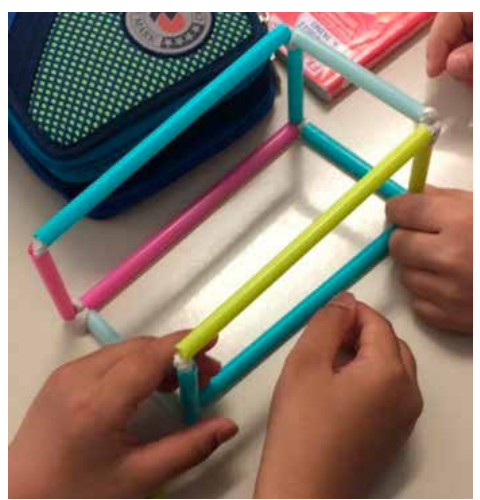

Figura 23. Costruzione del baule del mago Favoloso. 
Attraverso queste divertenti e partecipate attività, i bambini hanno imparato a riconoscere con sicurezza gli elementi dei solidi protagonisti (spigoli, facce e vertici) e a esserne coinvolti emotivamente, tanto da giocare con i protagonisti della storia attraverso la realizzazione di una drammatizzazione (Figure 24 e 25).

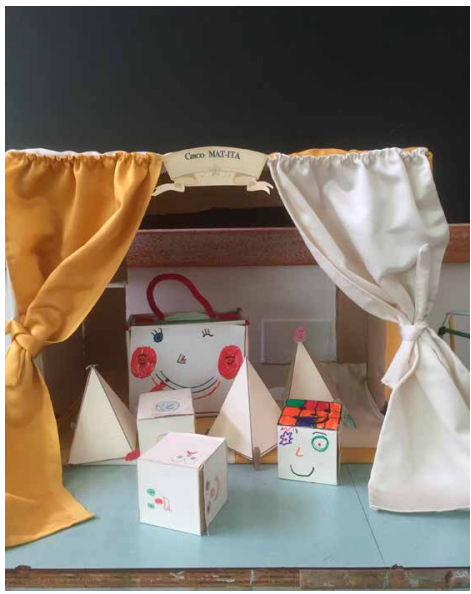

Figura 24. Plastico del circo Mat-Ita.

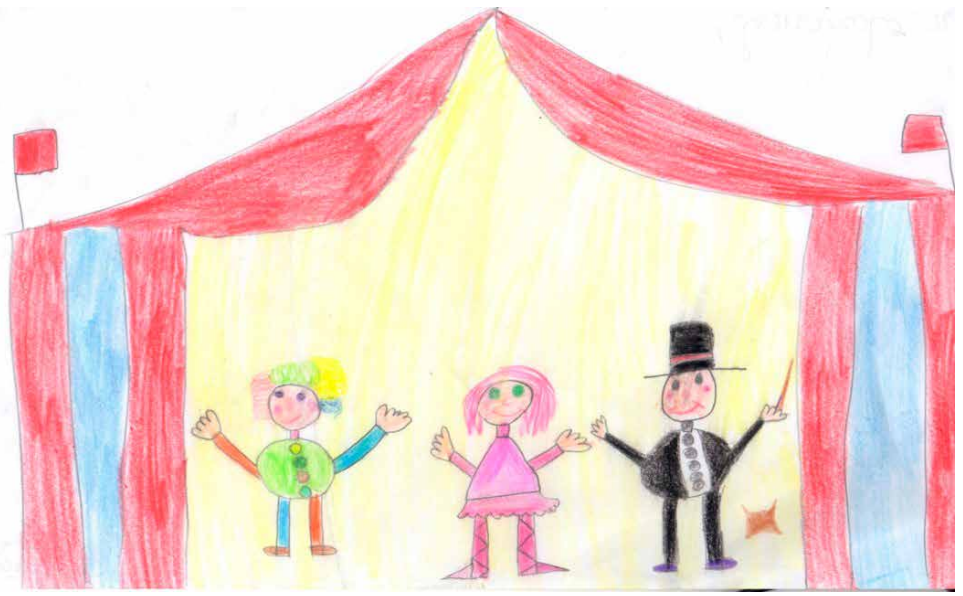

Figura 25. Rappresentazione grafica del circo Mat-Ita.

Un'altra storia scritta dal gruppo di insegnanti per condurre l'esplorazione delle figure geometriche piane con i bambini della scuola dell'infanzia è Filomena bimba curiosa... che con il filo segue ogni cosa. Anche in questo caso, è stato condiviso il percorso didattico, progettando insieme le attività, e ogni scuola ha poi realizzato degli albi, anche interattivi, utilizzando la creatività propria degli insegnanti e degli alunni (Figure 26 e 27).
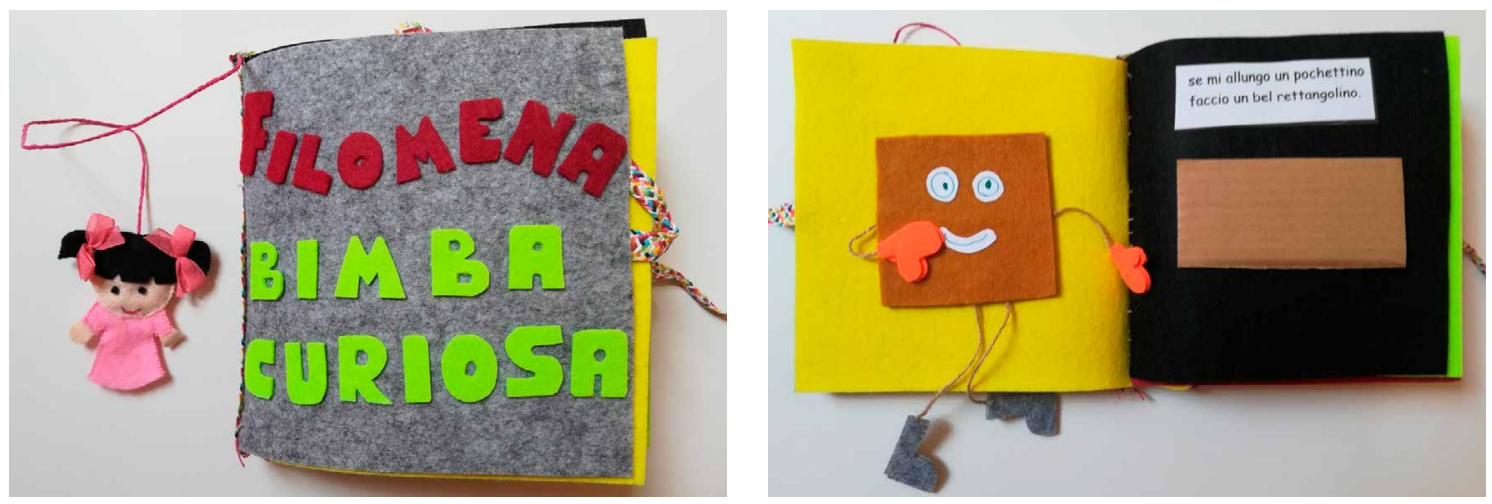

Figura 26. Filomena bimba curiosa (albo in pannolenci).

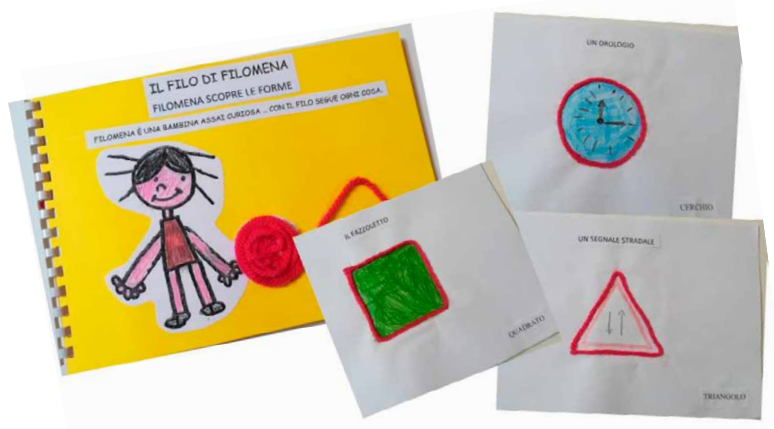

Figura 27. Filomena bimba curiosa (albo in carta). 
Prendendo spunto dall'ascolto della famosa canzone "Volta la carta" di Fabrizio De André, dopo aver esplorato le caratteristiche del cubo e i suoi possibili sviluppi, e usando la loro fantasia e creatività, gli alunni di una classe seconda primaria hanno interpretato gli 11 sviluppi del cubo e creato un bellissimo albo "Volta la carta e ... il cubo incarta" (Figure 28 e 29, Allegato 4) nel quale ogni sviluppo assume un significato.

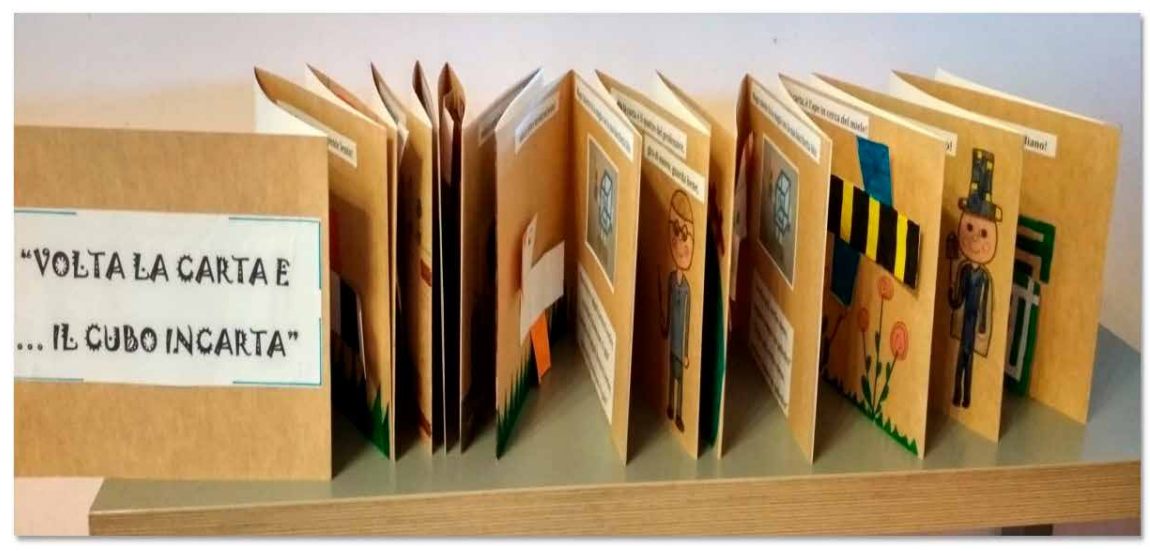

Figura 28. Albo illustrato "Volta la carta e ... il cubo incarta".
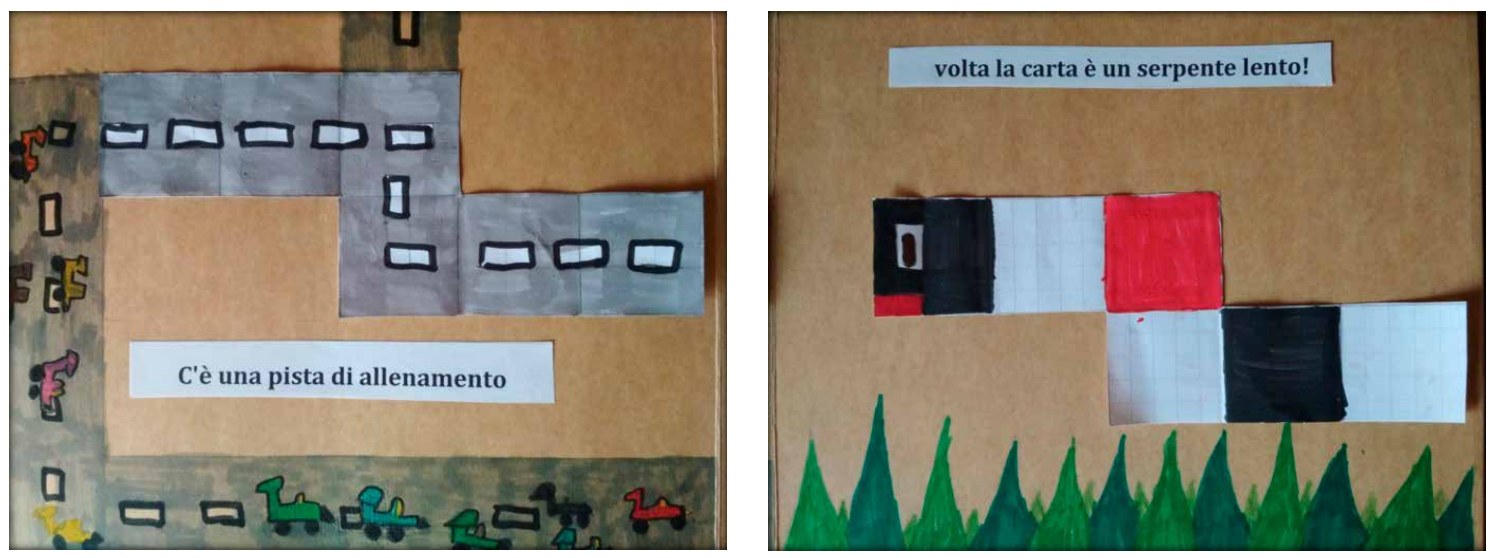

Figura 29. Esempi di alcune pagine dell'albo "Volta la carta e ... il cubo incarta".

Italmatica nelle storie. Nelle classi terze, quarte e quinte della scuola primaria abbiamo condotto delle riflessioni sulle parole del linguaggio specialistico della geometria e sulle interferenze con il loro uso nel linguaggio comune. Agli approfondimenti sul lessico, come già descritto nel paragrafo riguardante i problemi, abbiamo dedicato molta attenzione, perché conoscere e riconoscere correttamente il significato delle parole in contesto, e avere un ampio bagaglio lessicale, significa comprendere in modo profondo, costruire testi efficaci, avere maggiori possibilità di esprimere con chiarezza il proprio pensiero e riuscire a comunicare in modo efficace.

Sono state prodotte delle belle raccolte di immagini in libri cartacei e digitali, tipo dei dizionari illustrati con i diversi significati di parole polisemiche (Figure 30 e 31), e sono state prodotte delle interessanti storie che giocano sui doppi sensi ${ }^{6}$ (Figura 32).

6. Alcune delle storie sono anche reperibili al seguente link:

https://www.matematicando.supsi.ch/risorse-didattiche/intrecci-tra-matematica-e-italiano/. 

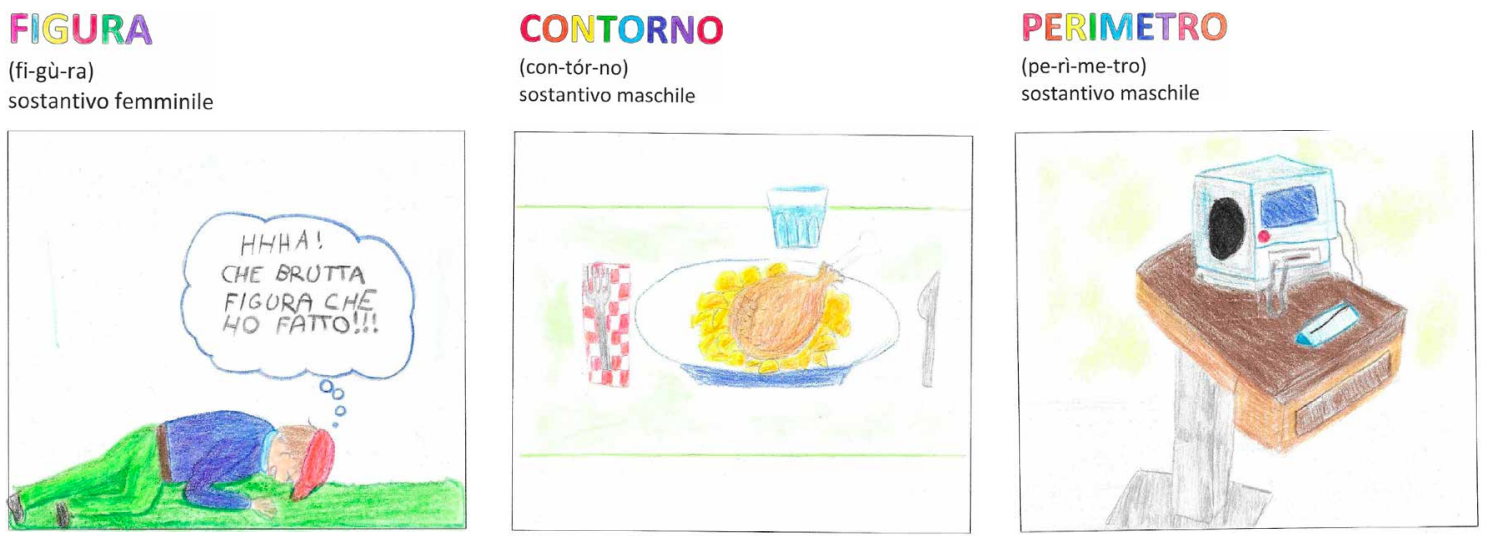

Figura 30. Esempi di alcune pagine del dizionario illustrato delle parole polisemiche.
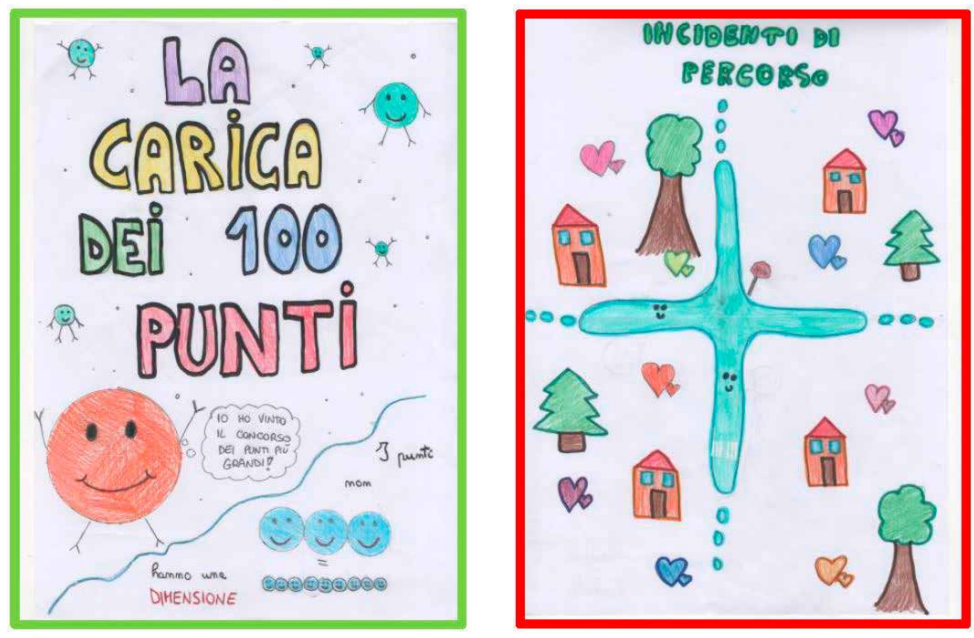

Figura 31. Copertine di alcune storie.
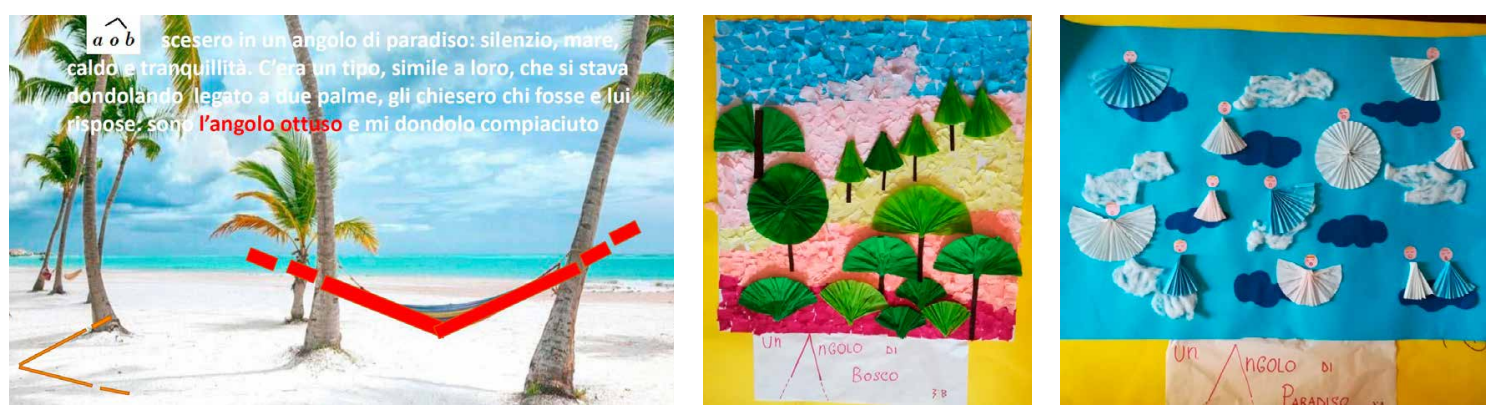

Figura 32. Lavori prodotti sulla storia "Antonietta e Benedetta negli angoli della terra"

Gli alunni di alcune classi terze e quarte della scuola primaria si sono cimentati nell'invenzione di storie unendo due figure costruite in precedenza con il tangram, seguendo il "binomio fantastico" teorizzato da Rodari (1973), ossia I'accostamento di due immagini, scelte casualmente, meglio ancora se abbastanza distanti fra loro. L'incontro/scontro tra le due immagini (le due parole) accostate crea il problema e mette in moto l'immaginazione degli alunni che, per creare una narrazione, devono trovare un punto di incontro in cui i due elementi estranei possano convivere (Figura 33). 


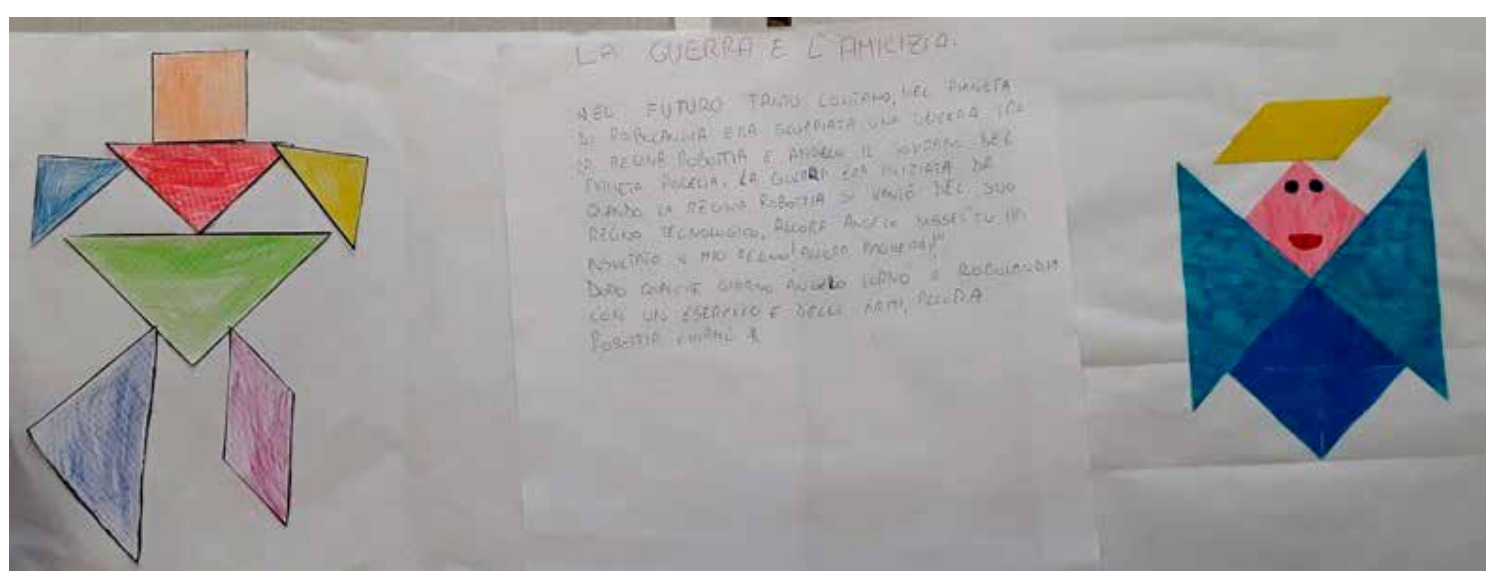

Figura 33. Il tangram e il "binomio fantastico".

Un'altra strategia per inventare storie è stata di prendere come spunto un lavoro artistico sulle linee: dopo aver disegnato, a caso, delle linee intrecciate su un foglio e colorato alcune regioni, gli alunni sono stati invitati a ritagliare alcune delle regioni individuate e, con le parti ritagliate, creare un nuovo disegno. In seguito, sono stati abbinati tre disegni scelti casualmente e, con essi, sono state inventate delle storie (Figura 34).

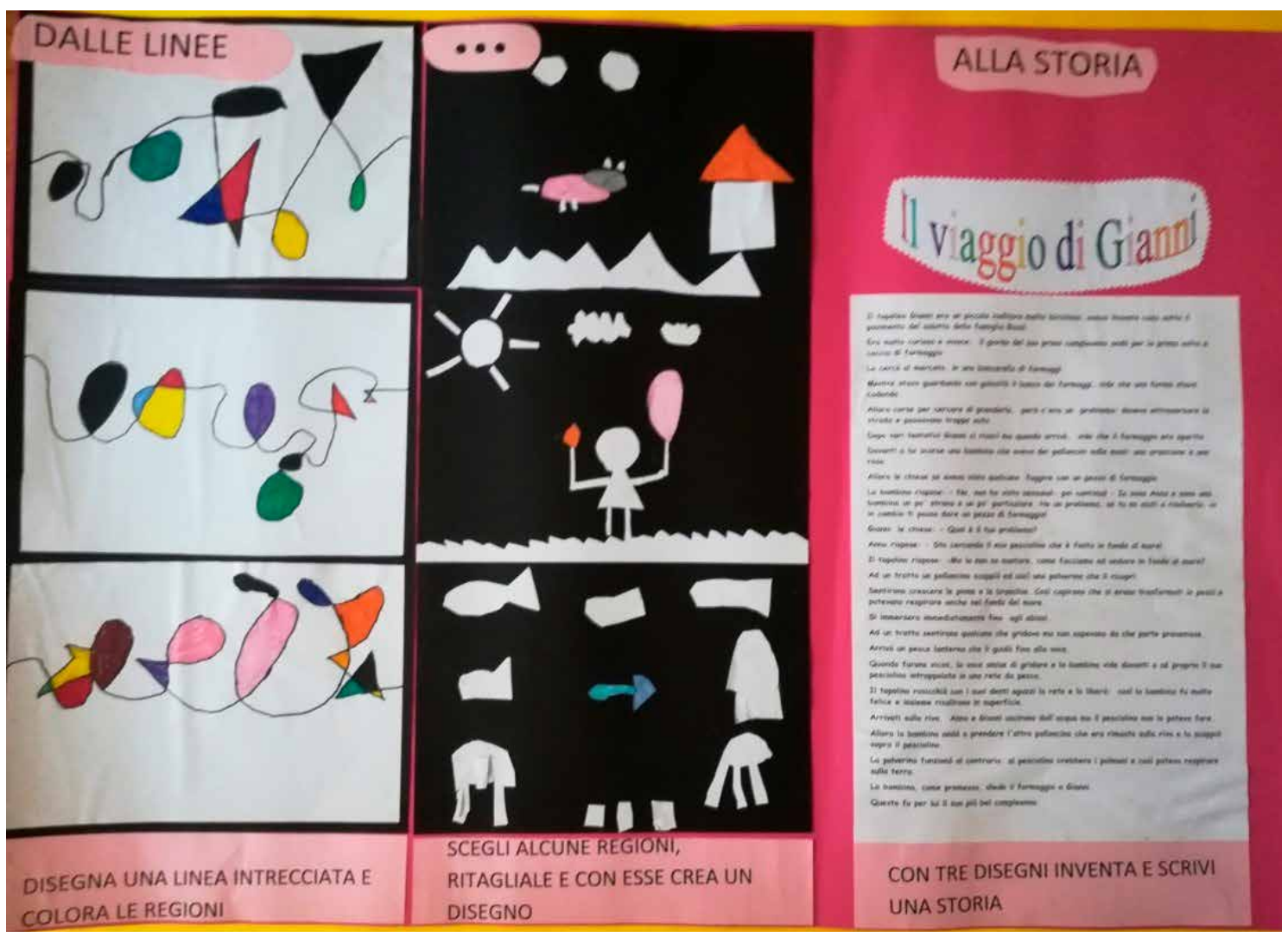

Figura 34. Dalle linee a una storia.

In questi lavori, è stata molto importante la collaborazione con gli insegnanti dell'area linguistica, con i quali gli alunni hanno prodotto tante storie, usando varie tecniche narrative, tra cui il giallo: il 
giallo di Carnevale, ad esempio, è un'avvincente avventura in cui l'investigatore deve scoprire chi ha "tagliato" in due Mister Rettangolo (Figura 35).

Con i compagni di classe crea dei gruppi di lavoro per risolvere...
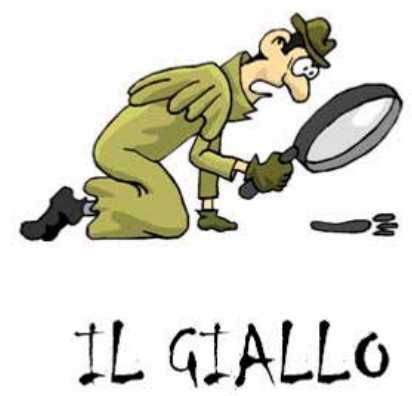

DI CARNEVALE...

Figura 35. Il testo giallo di Carnevale.
Alla festa di Carnevale erano invitati tutte le figure geometriche, tra cui Mister Rettangolo,...

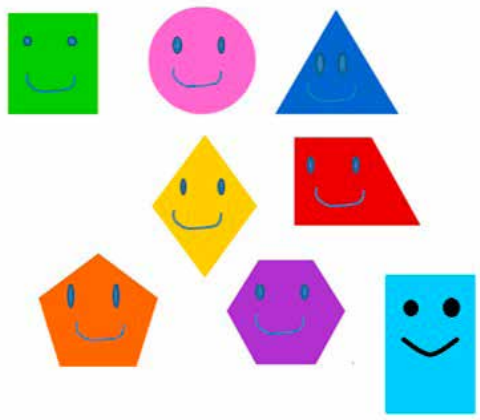

Argomentazione e storie. Abbiamo usato racconti e favole anche per allenare all'argomentazione. Argomentare è una capacità complessa di problem solving che presuppone operazioni cognitive e linguistiche specifiche e la capacità di mettersi nei panni dell'altro. È importante proporre ai bambini, fin dai primi anni della scuola primaria, attività specifiche e non occasionali creando una varietà di situazioni di confronto e scambio, soprattutto orale, in cui imparare a prendere la parola, a esporre e motivare le proprie idee e ad ascoltare le idee degli altri. L'argomentazione deve essere intesa come un atteggiamento nei confronti del sapere, di sé stessi e degli altri, che sviluppa il senso critico e la democrazia.

Con i più piccoli si può prendere spunto da attività quotidiane e, via via, anche attraverso le storie, $\mathrm{i}$ pretesti possono essere molteplici e adattati anche a testi matematici, come ad esempio i problemi. Gradualmente, oltre al lavoro orale, si può avviare la stesura di testi di opinione, ma cercando sempre di agganciarsi a situazioni coinvolgenti e che abbiano senso per gli alunni.

In terza primaria abbiamo proposto la lettura del libro "L'albero" di Shel Silverstein (2014) a conclusione del quale abbiamo chiesto di scrivere un breve testo rispondendo alla domanda: Secondo te chi si è comportato in modo corretto? L'albero o l'uomo? Dopo la stesura della risposta, i bambini si sono scambiati i testi e ciascuno ha letto ciò che aveva scritto il compagno; poi si è discusso in classe e ciascuno ha potuto esprimere i propri argomenti a sostegno di quanto aveva scritto. A conclusione della discussione, ciascuno ha avuto la possibilità di rivedere e/o ampliare la risposta data in precedenza. Tutti i loro testi, dopo la discussione, si sono arricchiti di nuovi elementi emersi durante la discussione che avevano condiviso e le loro argomentazioni sono diventate più ricche.

Nelle stesse classi è stata condotta un'esperienza analoga con alcune favole, come ad esempio quella della cicala e della formica. Invitati a scrivere chi secondo loro si era comportato in modo corretto, i bambini, in buona parte, avevano risposto che la formica era stata previdente, si era impegnata per trovarsi preparata per l'inverno e la cicala invece non aveva lavorato, aveva soltanto cantato, quindi meritava quel trattamento. Altri però, pur essendo d'accordo sul fatto che la formica aveva lavorato e si era preoccupata di mettere da parte il cibo per l'inverno, condannavano il suo egoismo e il fatto che avesse lasciato fuori al freddo la povera cicala, perché così sarebbe morta.

Prima della discussione orale, era stata proposta anche la lettura della seguente filastrocca di Rodari (1982): 


\author{
Alla formica \\ Chiedo scusa alla favola antica, \\ se non mi piace l'avara formica \\ io sto dalla parte della cicala \\ che il più bel canto non vende, regala.
}

Dopo una discussione animata, gli alunni che ritenevano che la formica avesse fatto bene a chiudere la porta in faccia alla cicala, in gran parte hanno accolto le argomentazioni dei compagni e alla fine, a parte pochissime eccezioni rimaste ferme sulla loro posizione, si sono trovati d'accordo sul fatto che, anche se la cicala non aveva lavorato e non si era preoccupata di nulla, la formica avrebbe dovuto accoglierla nella sua tana perché lasciandola morire anche lei avrebbe sbagliato.

Analoghe attività si possono svolgere con i testi dei problemi. Le discussioni sono sempre molto interessanti e, in sostanza, fanno emergere sempre il buon senso dei bambini. Svolgendo con regolarità esperienze di questo tipo, sia con le storie, sia, con i problemi, in un clima sereno, aperto e accogliente, gli alunni si abituano al confronto, a esprimere il loro pensiero, le loro idee e questo contribuisce allo sviluppo della capacità di ascolto e allo sviluppo del senso critico.

\title{
3.3 Matematica e ludolinguistica
}

II gioco ha avuto sempre molto spazio nelle nostre pratiche didattiche, perché è uno strumento fondamentale per dare significatività all'apprendimento sia in ambiente reale, sia in ambiente digitale. Nel gioco ci sono regole da rispettare, comunicazioni da fare, finalità ben precise, strategie da mettere in campo, pertanto è uno dei contesti più adatto per gli alunni di tutte le età. Giocando, sia i bambini, sia i ragazzi, sono più rilassati, più attenti e più propensi alla socializzazione. II gioco, in sé, è uno degli strumenti più efficaci per attivare la comunicazione, la discussione, l'argomentazione e la negoziazione di significati. È divertente, motivante e consente un approccio positivo all'errore: se qualcosa non va si ricomincia, senza troppe frustrazioni e senza vivere la situazione come un fallimento. Come cita Bartezzaghi (2001), Umberto Eco aveva evidenziato l'importanza del gioco, mettendolo al quarto posto tra i bisogni fondamentali dell'uomo, dopo il nutrimento, il sonno, I'affetto e prima ancora del chiedersi il perché delle cose. II gioco, in tutte le sue varie tipologie e forme, è uno strumento formidabile per veicolare apprendimenti.

Nel caso specifico, la ludolinguistica, cioè «quella parte di linguistica che si occupa di combinazioni lessicali, di giochi di parole ecc.» (De Mauro, 2007), applicata alla matematica costituisce un campo di esplorazione molto creativo e stimolante. Lo spunto iniziale fornito dalle docenti Sbaragli e Demartini, partiva dalle proposte del maestro enigmista Francesco Giudici, detto "Ceck". Il libro "Giocare con le parole" (Fornara \& Giudici, 2016), del quale è coautore insieme a Simone Fornara, presenta una vasta gamma di giochi linguistici che possono essere presi come esempi per inventarne altri con le parole della matematica. Oltre a questo testo, abbiamo fatto riferimento anche ai libri "I Draghi locopei" (Zamponi, 1986) e a "Grammatica della fantasia" (Rodari, 1973).

Ci siamo divertiti tra noi docenti e con gli alunni a giocare, a costruire e inventare giochi nei quali protagoniste sono le parole: cruciverba, tautogrammi, abbinamenti fantastici, giochi di parole e geometria, crucipuzzle, mesostici, rebus, anagrammi, anche usando software tra i tanti disponibili online. ${ }^{7}$

Cruciverba. II gruppo di docenti ha costruito tanti cruciverba: alcuni molto semplici, utilizzati, ad esempio, per ripassare le tabelline in seconda primaria (Figura 36), altri, più complessi perché giocano 
sui doppi sensi di termini specialistici, inventati con gli alunni di quinta primaria (Figura 37) e della classe prima della scuola secondaria di primo grado (Figura 38).

Tabellina del 7

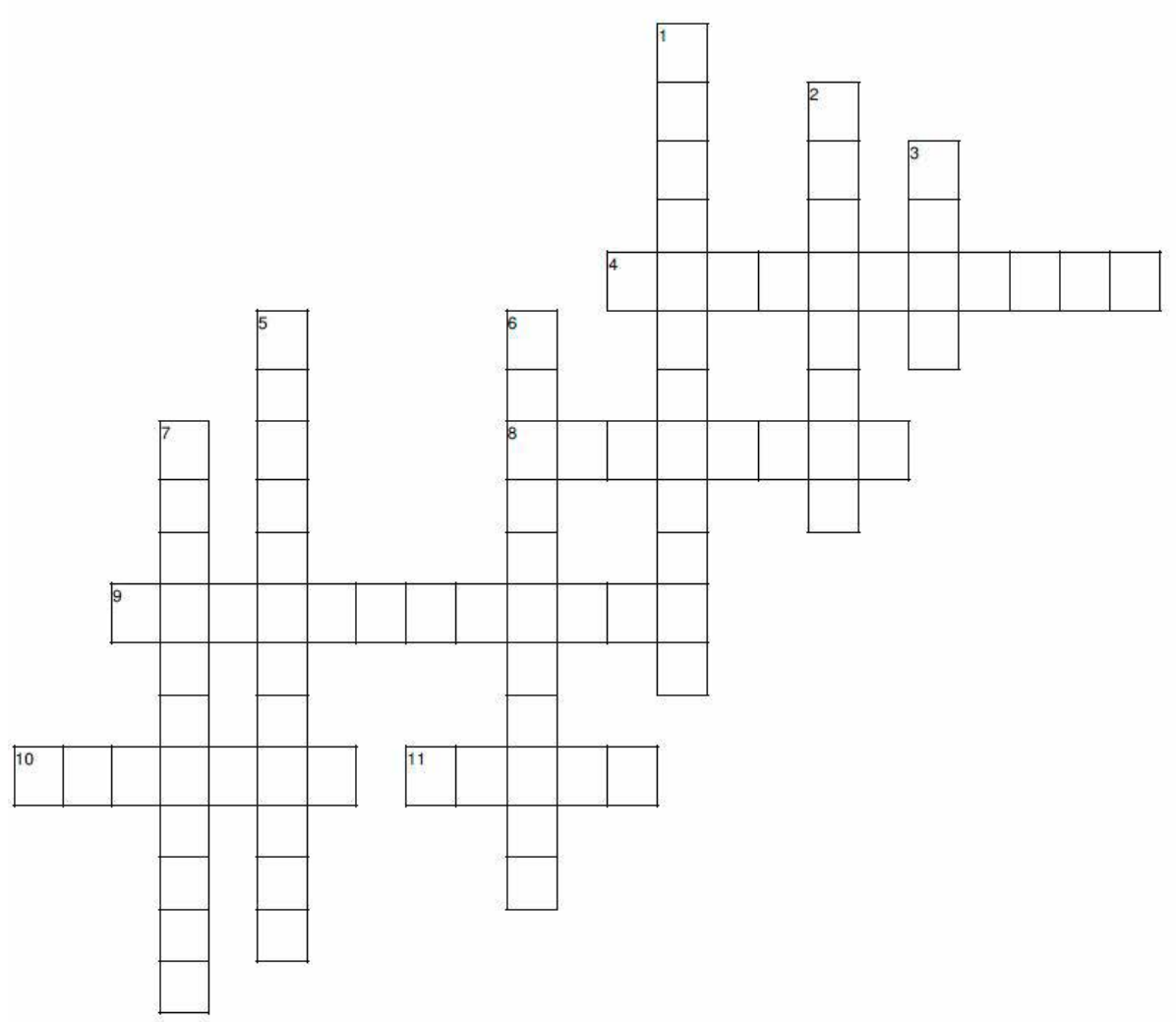

Across

4. $7 \times 2=$

8. $7 \times 10=$

9. $7 \times 5=$

10. $7 \times 3=$

11. $7 \times 1=$

Down

1. $7 \times 8=$

2. $7 \times 4=$

3. $7 \times 0=$

5. $7 \times 7=$

6. $7 \times 9=$

7. $7 \times 6=$

Figura 36. Cruciverba tabellina del 7.

\section{$\underset{\text { geometria }}{\text { doppio senso }}$}

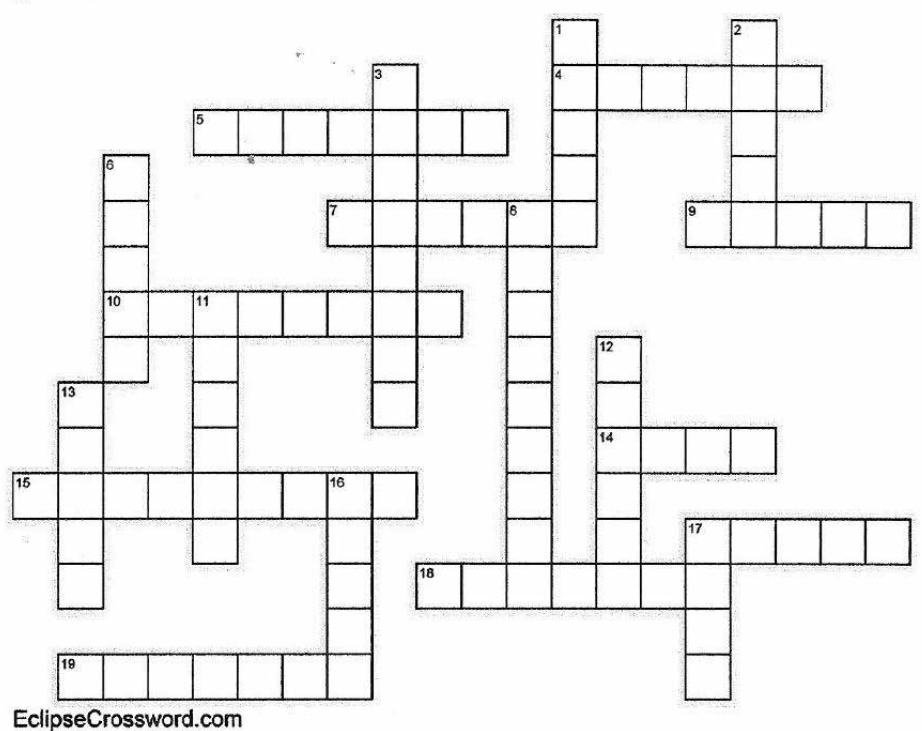

\section{Across}

4. Farne una brutta ... non è piacevole.

5. Al ... del nostro Istituto c'è il Dirigente.

7. Qualcuno ce l'ha tosta.

Quello dei motori è fastidioso.

10. Al circo vi si esibiscono gli acrobati.

14. Lì dentro il portiere può usare le mani.

15. Attrezzo per la ginnastica.

17. Tratto di strada non diritta.

18. I bambini devono fare attenzione a non sbattere la testa in quello del tavolo.

19. Sua ... reale.

\section{Down}

1. Si può scrivere con la penna a

C'è anche quello di parentela.

Nell'antico Egitto era una tomba.

Durante la prova di evacuazione tutti vanno

verso quello di raccolta.

Li provocano i veicoli che si scontrano.

11. $E^{\prime}$ un calcio che si batte quando rimette in gioco la squadra avversaria.

Ci si va con il razzo.

13. Si suona e ha i tasti bianchi e neri.

16. C'è quella telefonica.

17. Quello gelato si gusta d'estate.

Figura 37. Cruciverba "doppio senso". 

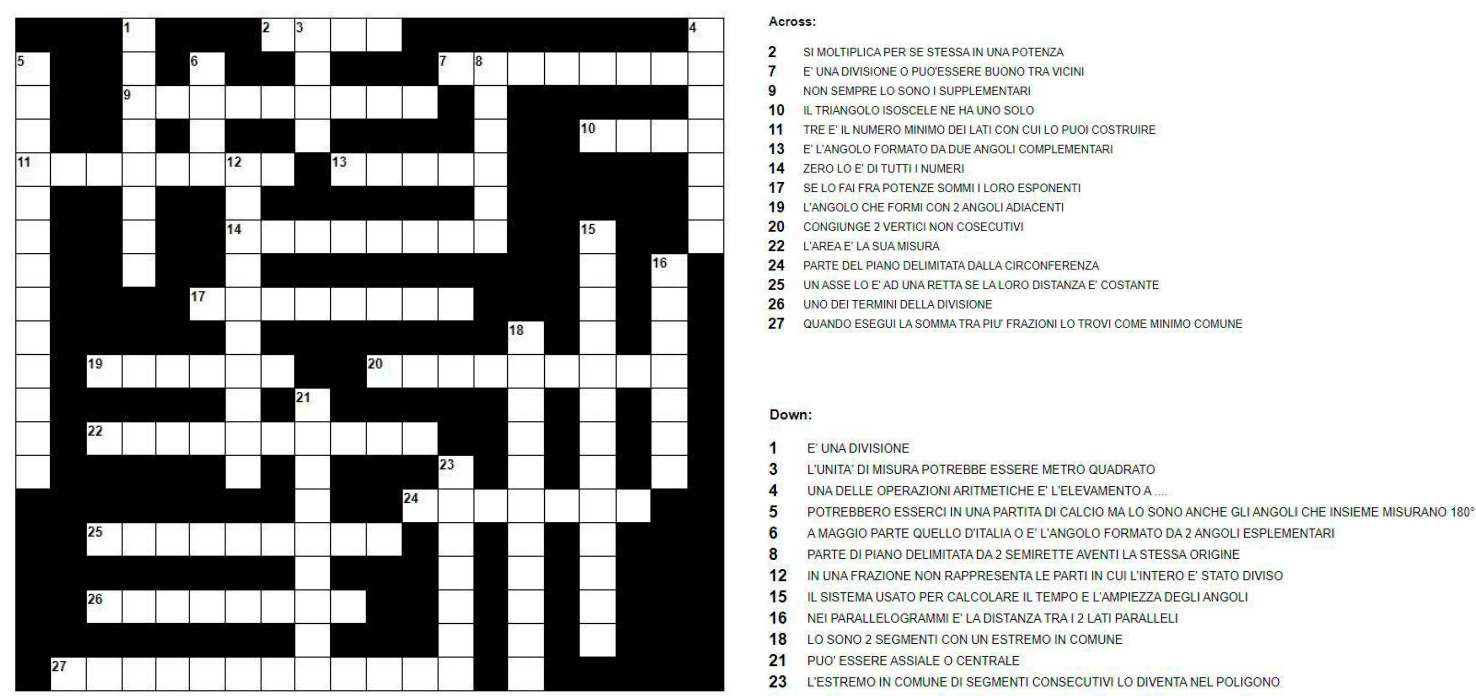

Figura 38. Cruciverba "incroci matematici"

Tautogrammi. In diverse classi abbiamo giocato con i tautogrammi, cioè frasi in cui tutte le parole iniziano con la stessa lettera. In classe seconda, dopo aver letto insieme "Alfabetiere" (Munari, 1998) e "LABICl. 26 lettere in gioco" (Piumini, 2010), abbiamo realizzato uno speciale abbecedario (Allegato 5) nel quale le lettere dell'alfabeto sono costruite con il tangram e ad ogni lettera è abbinata una frase elaborata con la tecnica del tautogramma (Figura 39).
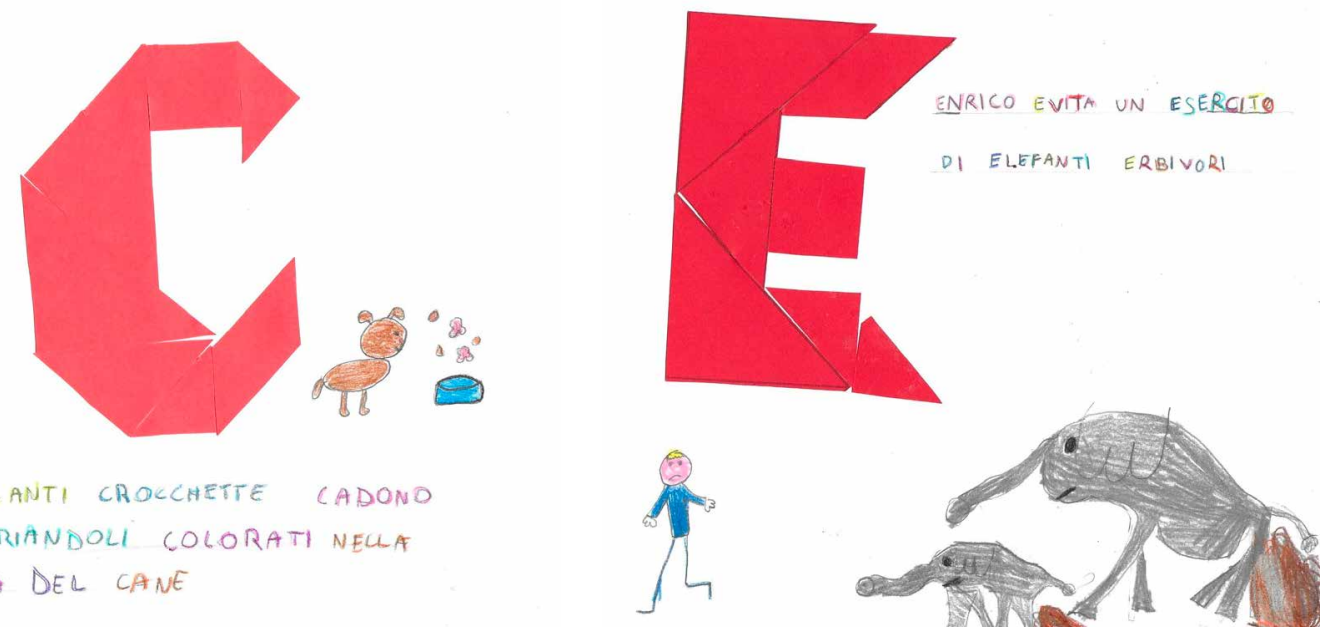

Figura 39. Due pagine dell'Abbecedario realizzato con il tangram.

Tautogrammi geometrici, con i nomi di figure piane o solide, sono stati inventati e illustrati dalla seconda primaria e fino alla secondaria di primo grado. Con essi sono stati realizzati dei libri in formato cartaceo per la biblioteca della scuola e digitale per tutti gli alunni (Figure 40, 41 e 42). In genere i più piccoli sono stati lasciati liberi di creare con l'unico vincolo di usare almeno tre parole; i più grandi invece si sono cimentati con vincoli maggiori. In alcuni casi, ad esempio, dovevano scegliere il nome di una figura geometrica, un aggettivo e un verbo e con essi comporre un testo in cui tutte le parole avessero la stessa iniziale, ad eccezione degli articoli e delle preposizioni; in altri casi, invece, veniva assegnato come vincolo il numero minimo di cinque o sette parole, tutte con la stessa iniziale, per 
comporre la frase. I bambini all'inizio erano un po' spaesati, ma, dopo le prime produzioni, si sono divertiti molto sfidandosi a chi riusciva a trovare le frasi più divertenti e più lunghe. Ecco alcuni esempi: ${ }^{8}$

- Una sfera sincera salta con il serpente.

- Pentagono penoso, patetico, parte piangendo.

- Un cono carino consolava il cerchio col caschetto caduto dal cornicione della casa del cubo.

- I poligoni parlarono con Pitagora per portare panini e pizze ai poveri.

- II trapezio e il triangolo tornavano a Torino con il trattore. II trattore talvolta travolgeva i tassi. II triangolo telefonò al taxi.

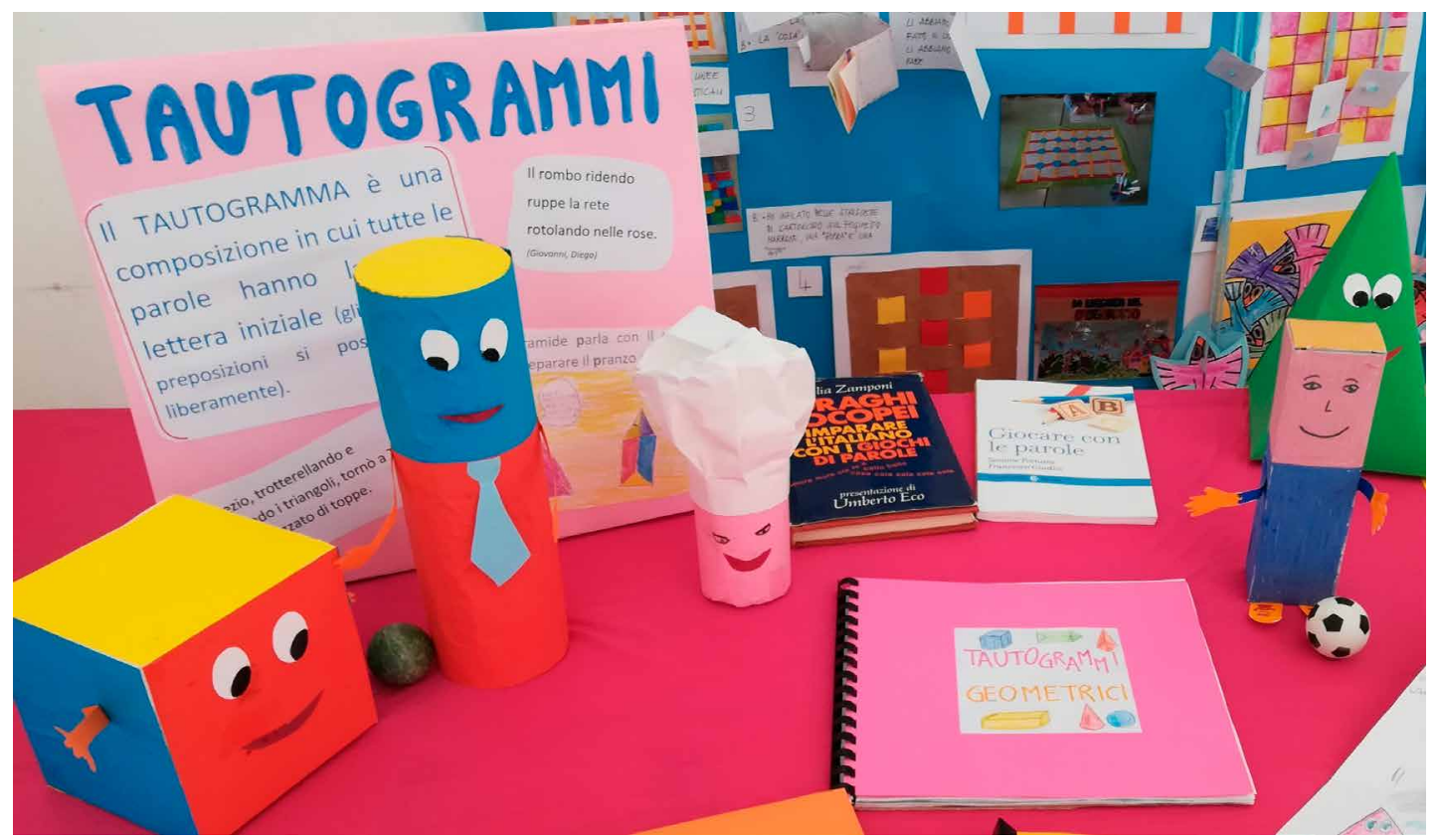

Figura 40. Personaggi e raccolte dei tautogrammi.

\section{Cubo Carlo compra un cocomero croccante con il Conte Cilindro.}

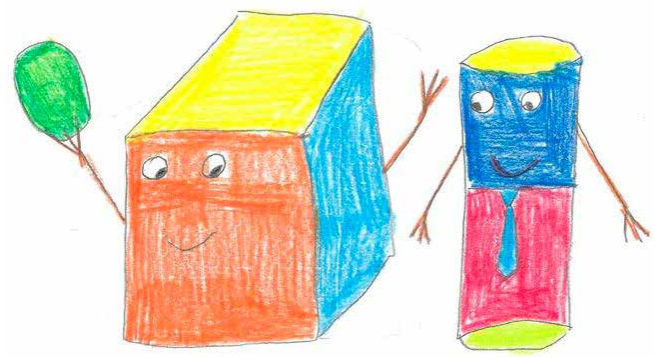

Figura 41. Esempi di tautogrammi di seconda primaria.

8. Altri tautogrammi sono reperibili all'indirizzo: https://www.matematicando.supsi.ch/risorse-didattiche/intreccitra-matematica-e-italiano/. 

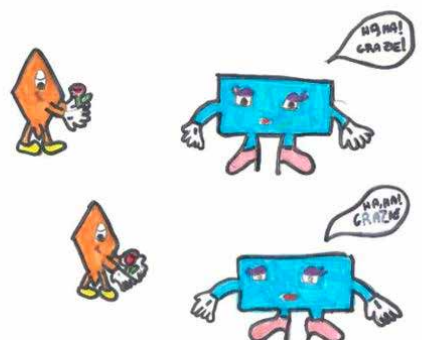

I rombi regalarono rose rosse ai rettangoli che, ridendo, li ringraziarono.
II rombo ridendo

ruppe la rete

rotolando nelle rose.

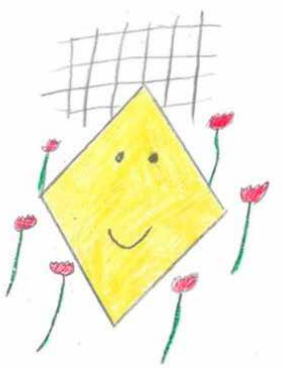

Figura 42. Esempi di tautogrammi di quinta primaria.

Rebus. Giocare con i rebus e inventarne per sfidare gli amici sono attività che abbiamo proposto a partire dalla classe seconda fino alla quinta della scuola primaria. I bambini hanno costruito e disegnato rebus con numeri o con figure geometriche o con alcune loro caratteristiche. Sono stati prodotti su cartoncini formato cartolina e gli alunni si sono divertiti sfidandosi nella ricerca di soluzioni e scambiandoli con alunni di altre classi.

Riportiamo alcuni esempi tratti dalla raccolta dei rebus di classe quinta (Figura 43).
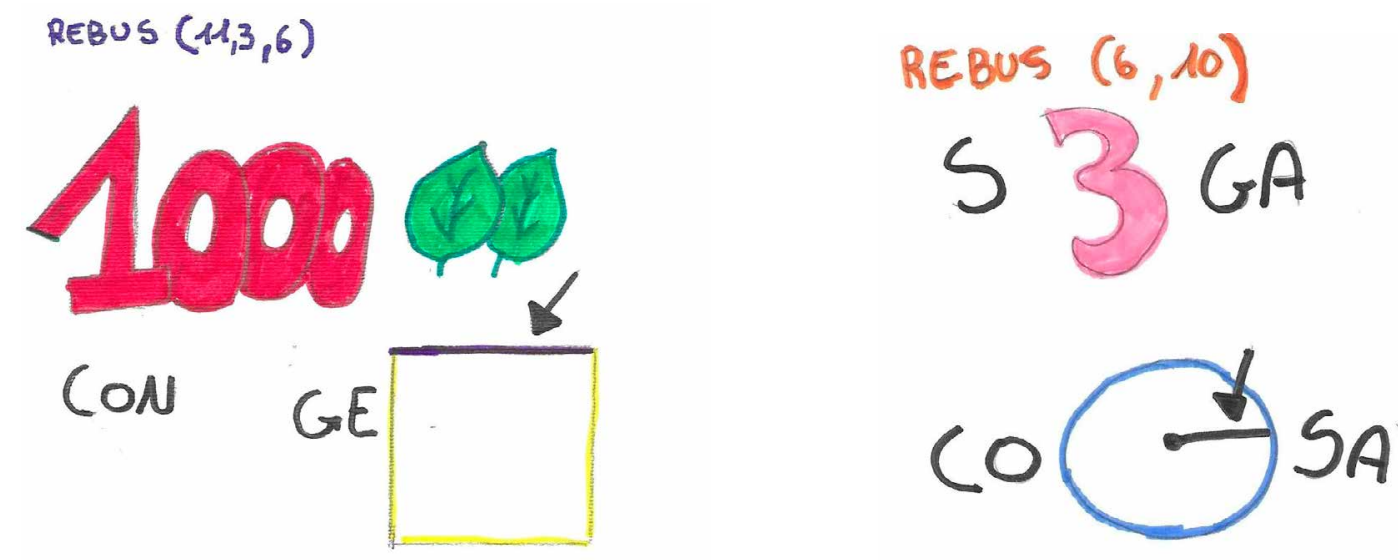

Figura 43. Esempi di rebus inventati in classe $\mathrm{V}$ primaria.

Anagrammi. Anche con gli anagrammi sono state compiute interessanti esperienze. L'anagramma è uno dei giochi enigmistici più diffusi, ma non è molto facile, perché presuppone solide abilità di scrittura e conoscenze ed esperienze abbastanza ampie. La valigia dei numeri (Figura 44) è un gioco costruito in classe quinta primaria; si tratta di un gioco da tavolo in cui ci si sfida, divisi in due squadre, a suon di anagrammi delle parole dei numeri. Nella valigia sono contenuti tutti i materiali: due mazzi di carte anagrammi; le istruzioni del gioco; il tabellone per registrare i punteggi.

Costruire le carte per il gioco (alcuni esempi nelle Figure 45 e 46), inventare e scrivere le regole per giocare, stabilire il numero di giocatori sono attività che hanno richiesto un lavoro notevole di problem solving, di confronto e di ricerca; hanno richiesto inoltre una particolare cura nella comunicazione e una scrittura chiara ed efficace, ottenuta dopo diverse revisioni sollecitate dallo scambio delle prime stesure dei testi tra i diversi gruppi di lavoro. 


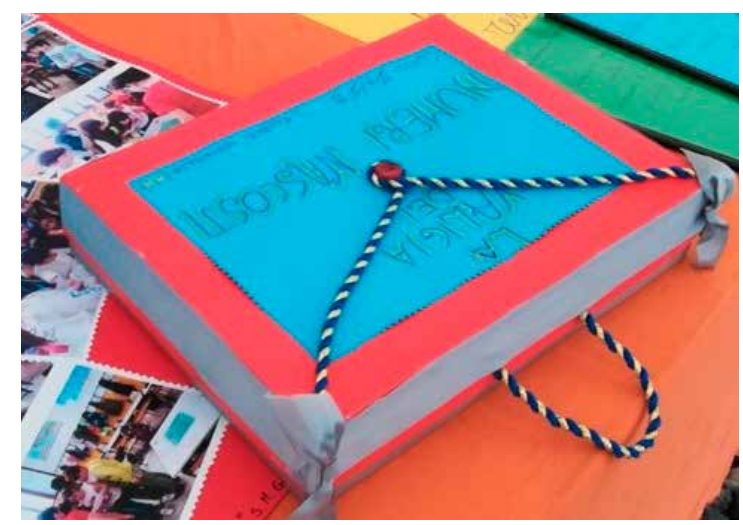

Figura 44. La valigia dei numeri.

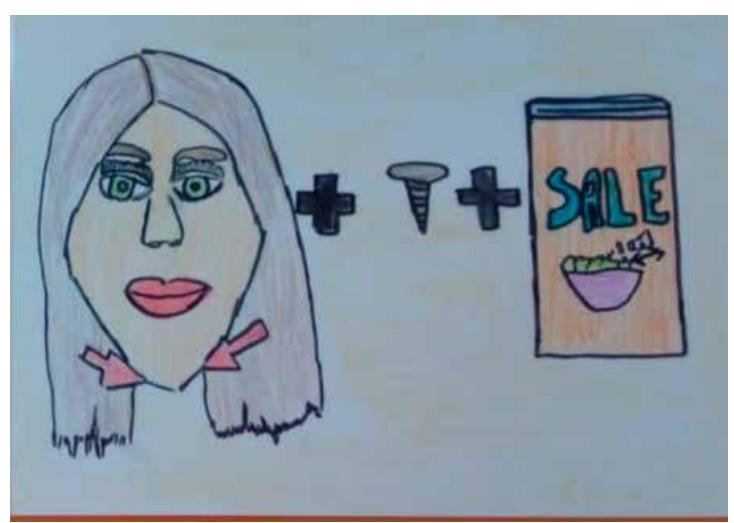

Figura 45. Anagramma: Mento + vite + sale $\rightarrow$ Novemilasette

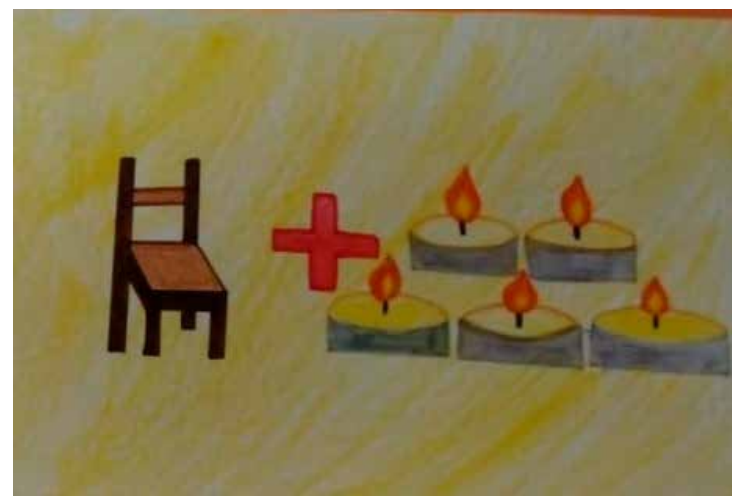

Figura 46. Anagramma: Sedia + lumicini $\rightarrow$ Seimilaundici.

"Dove ci incontriamo?". Un interessante gioco, divertente e utile per approfondire le conoscenze fondamentali delle figure geometriche, è "Dove ci incontriamo?". In questo gioco, che prende spunto dal binomio fantastico di Rodari, si deve trovare un elemento comune a due figure estratte a sorte. Gli alunni hanno preparato alcuni foglietti su ciascuno dei quali hanno scritto il nome di una figura geometrica piana o solida; i foglietti sono stati piegati e posti in una scatola. Nel momento del gioco venivano estratti due foglietti alla volta e i due nomi dovevano essere abbinati cercando di trovare qualcosa in comune. Dopo aver ascoltato tutte le proposte, insieme hanno scelto quella che ritenevano migliore. Per rimanere nel contesto fantastico iniziale, le figure sono state personificate ed è scaturita una raccolta di punti di incontro, originale e creativa. Alcuni esempi nella Figura 47.
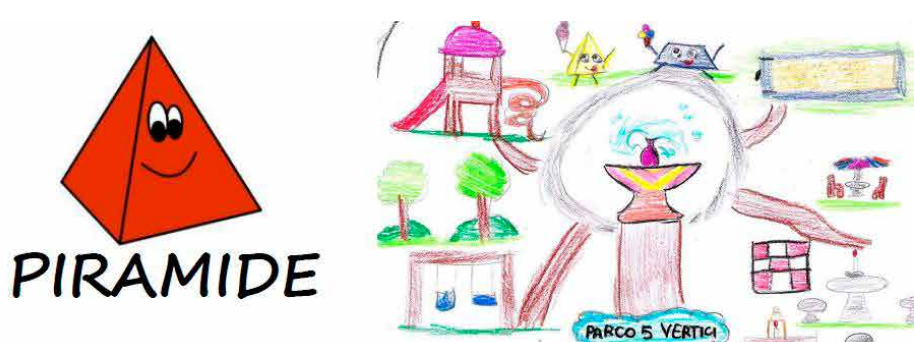

\section{PENTAGONO}

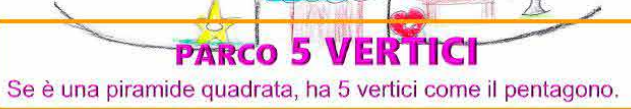



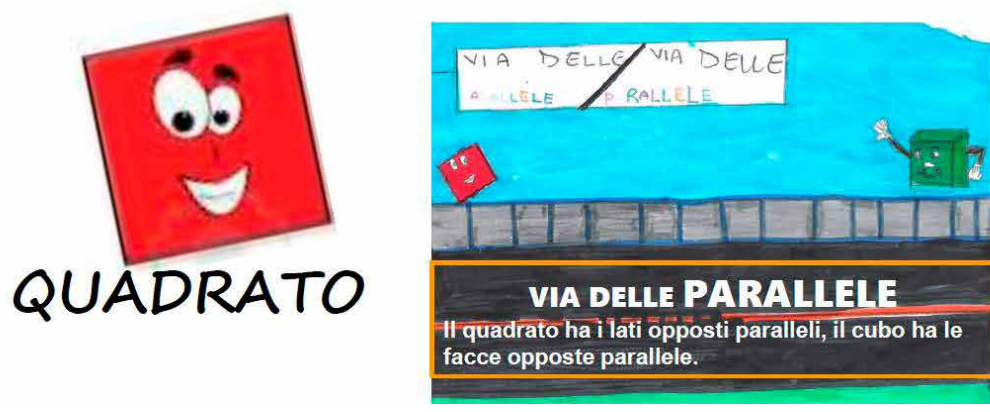

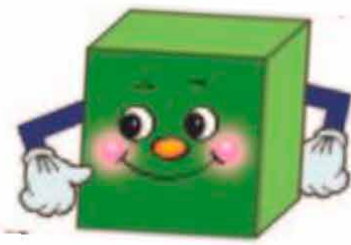

CUBO

Figura 47. Alcuni esempi del gioco "Dove ci incontriamo?"

Mesostico. Un altro gioco linguistico di cui abbiamo scoperto la grande efficacia didattica è il mesostico. II mesostico è un'alternativa all'acrostico, solo che invece della lettera iniziale, quella su cui ci si concentra è una lettera situata nella parte centrale della parola.

L'attività ha coinvolto ragazzi delle classi quinte primaria e delle classi prime della scuola secondaria di primo grado. Dopo aver mostrato qualche esempio e spiegato le regole di questo gioco linguistico non molto conosciuto, la classe è stata divisa in due gruppi, a ciascun gruppo è stata fornita la griglia riempita e i ragazzi sono stati invitati a scrivere le definizioni (Figura 48); in qualche classe i ragazzi hanno costruito anche la griglia.

\section{MESOSTICO}

Cl. 5 - gruppo 1 SCRIVI LE DEFINIZIONI

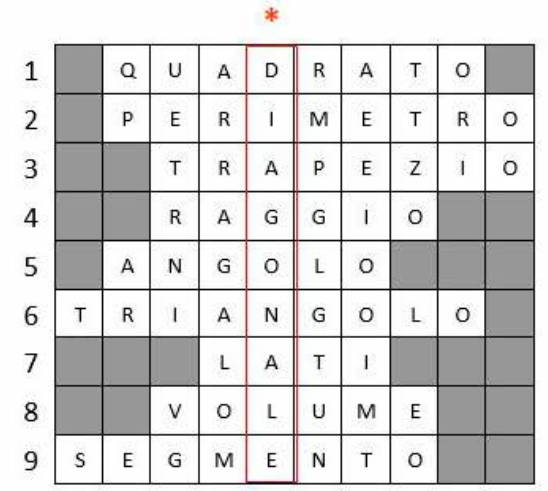

ORIZZONTALI

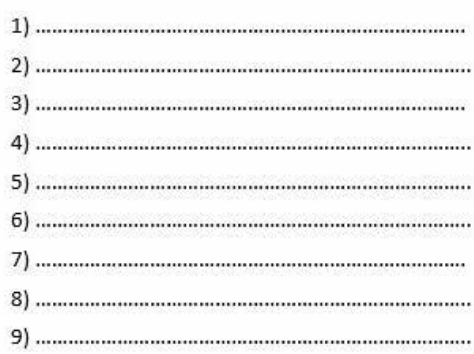

VERTICALE

*
MESOSTICO

cl. 5 - gruppo 1

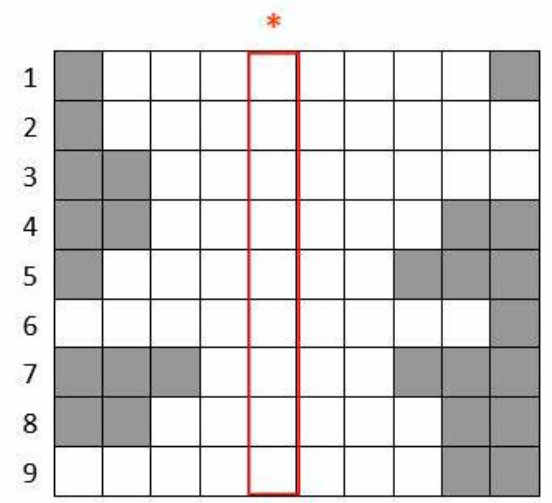

ORIZZONTALI

1) Un poligono regolare.

2) Misura del confine di una figura.

3) Può essere scaleno.

4) Parte di una bicicletta.

5) Si misura in gradi.

6) Può essere acutangolo, rettangolo, ottusangolo.

7) Un quadrilatero ne ha 4.

8) Lo alzi nella radio.

9) Parte di una retta.

\section{VERTICALE}

* 
Completato il lavoro, i due gruppi si sono scambiati le definizioni che sono state consegnate unitamente alla griglia vuota: quella del gruppo 1 è stata consegnata al gruppo 2 e viceversa (Allegato 6). Questo gioco è risultato molto interessante, perché ha consentito di riflettere sulla comunicazione in geometria, su che cosa è necessario e sufficiente per individuare il nome di una figura o una caratteristica precisa.

Nella discussione che nasce a conclusione del gioco vengono inevitabilmente contestate le definizioni dei compagni non riconosciute comprensibili o valide, e dunque vengono sollecitate le capacità argomentative, dato che viene chiesto ai ragazzi di motivare le loro contestazioni o le giustificazioni delle loro scelte.

Quelli che abbiamo descritto sono alcuni esempi di attività ludolinguistiche che abbiamo trasformato in giochi italmatici. Nello svolgere queste attività, ci siamo più che mai convinti che giochi di enigmistica linguistico-matematici si possono proporre anche nei primi anni della scuola primaria, perché i bambini si appassionano nella scoperta delle parole, delle loro trasformazioni e dei loro significati. Questi giochi hanno una grande valenza didattica: favoriscono l'ampliamento lessicale e I'uso appropriato di termini specifici; favoriscono il passaggio da un uso spontaneo e naturale della lingua a una consapevolezza ragionata; favoriscono l'acquisizione di concetti matematici e potenziano e sviluppano le competenze comunicative. Spiegare le regole di un gioco ai compagni, inoltre, richiede una comprensione profonda, un grande sforzo comunicativo e aiuta a mettere in ordine le proprie idee. Infine, proprio perché si tratta di giocare, la motivazione e la partecipazione degli alunni sia nello svolgimento sia nell'invenzione dei giochi sono state sempre molto elevate, instaurando così un clima piacevole e positivo.

\section{Bibliografia}

Arrigo, G., \& Sbaragli, S. (2004). I solidi. Riscopriamo la geometria. Carocci.

Bartezzaghi, S. (2001). I diritti del/'Homo ludens. https://ricerca.repubblica.it/repubblica/archivio/repubblica/2001 101/06/diritti-dell-homo-ludens.html.

Cottino, L., \& Sbaragli, S. (2005). Le diverse "facce" del cubo. Carocci.

Dahl, R. (2016). Matilde. Salani.

Demartini, S., \& Sbaragli, S. (2015). Geometria e narrazione alla scuola dell'infanzia: un "binomio fantastico". In B. D’Amore \& S. Sbaragli (A cura di), La didattica della matematica, disciplina per l'apprendimento (pp. 6772). Pitagora.

Demartini, S., Fornara, S., \& Sbaragli, S. (2017). Numeri e parole. Giunti.

Demartini, S., \& Sbaragli, S. (2019). La porta di entrata per la comprensione di un problema: la lettura del testo. Didattica della matematica. Dalla ricerca alle pratiche d'aula, 5, 9-43. https://doi.org/10.33683/ddm.18.5.1

De Mauro, S. (2007). Gradit (Grande dizionario italiano della lingua dell'uso). Utet.

Fornara, S., \& Giudici, F. (2016). Giocare con le parole. Carocci.

Fornara, S., \& Sbaragli, S. (2013). Italmatica. Riflessioni per un insegnamento/apprendimento combinato di italiano e matematica. In B. D’Amore \& S. Sbaragli (A cura di), La didattica della matematica come chiave di lettura delle situazioni d'aula (pp. 33-38). Pitagora. 
Un percorso integrato di matematica e italiano in continuità dalla scuola dell'infanzia alla scuola secondaria di primo grado / Lorella Campolucci e Danila Maori

Gramsci, A. (2019). L'albero del riccio. Iskra.

Lingdren, A. (2010). Pippi Calzelunghe. Salani.

Ministero dell'istruzione, dell'università e della ricerca. (2018). Indicazioni nazionali e nuovi scenari. https:// www.miur.gov.it/documents/20182/0/Indicazioni+nazionali+e+nuovi+scenari/

Munari, B. (1998). Alfabetiere. Corraini.

Piumini, R. (2010). LABICI. 26 lettere in gioco. Artebambini.

Rodari, G. (1973). Grammatica della fantasia. Einaudi.

Rodari, G. (1982). Filastrocche in cielo e in terra. Einaudi.

Silverstein, S. (2014). L'albero. Salani.

Zamponi, E. (1986). I draghi locopei. Imparare l'italiano con i giochi di parole. Einaudi. 